\title{
Numerical Simulation of Mixing Enhancement in a Hot Supersonic Jet
}

\author{
C.E. Grosch \\ Departments of Oceanography and Computer Science \\ Old Dominion University, Norfolk, Virginia 23529 \\ J.M. Seiner \\ Aeroacoustics Branch \\ NASA Langley Research Center, Hampton, VA 23681 \\ M.Y. Hussaini \\ Program in Computational Science and Engineering \\ Florida State University, Tallahassee, FL 32306 \\ T.L. Jackson \\ Institute for Computer Applications in Science and Engineering \\ NASA Langley Research Center, Hampton, VA 23681
}

\begin{abstract}
Experimental observations show that the presence of small tabs on the edge of a hot, compressible jet exiting into a slower moving, colder ambient flow can increase the rate of spreading of the jet. This suggests that the rate of mixing of the jet and the ambient fluid is also increased. In order to elucidate the physical mechanism responsible for the increased spreading rate a set of calculations were carried out within the framework of the compressible three dimensional Navier-Stokes equations. A series of grid refinements were made to assess the accuracy of the results. We first simulated the flow without the tabs, obtaining reasonable agreement with experimental measurements of the velocity. We then simulated the flow, without tabs, over a range of values of the convective Mach number in order to determine the dependence of the mixing on this parameter. Simulations with modeled tabs were also carried out. In these calculations the effect of the tabs on the flow was modeled by pairs of counter rotating vortices. The results of these calculations indeed show that the presence of the tabs increase the spreading rate of the jet. The basic physical mechanism responsible for the enhanced spreading rate is discussed and qualitative comparisons with flow visualizations are made.
\end{abstract}

The first, third and fourth authors were supported by the National Aeronautics and Space Administration under NASA Contract No. NAS1-19480 while in residence at the Institute for Computer Applications in Science and Engineering (ICASE), NASA Langley Research, Hampton, VA 23681-0001. 


\section{Introduction}

Two programs have been initiated by NASA that have identified engine noise reduction as an enabling technology. These programs are the NASA High Speed Research (HSR) and Advanced Subsonics Technology (AST) programs. In the HSR program, jet noise is the principle contributor. In the AST program, jet noise is the principle contributor for aircraft in the current fleet, where a goal of $3 \mathrm{~dB}$ reduction is established for aircraft engines with bypass ratios up to 5 . Methods used to reduce jet noise in both programs often utilize concepts that enhance mixing between high and low speed streams (Seiner and Krejsa ${ }^{[1]}$ ). Of these, the most popular methods utilize concepts that introduce streamwise axial vorticity. Often this is accomplished through the use of a lobed mixer with ejector (Presz ${ }^{[2]}$; Tillman, Patrick and Peterson ${ }^{[3]}$ ). Other methods utilize tab-like devices to generate a pair of counter-rotating axial vortices to enhance stream mixing (Ahuja and Brown ${ }^{[4]}$; Samimy, Zaman and Reeder ${ }^{[5]}$; Zaman ${ }^{[6]}$; Ahuja ${ }^{[7]}$; Zaman, Reeder and Samimy $\left.{ }^{[8]}\right)$.

Although it has been known for a number of years that tabs can have a substantial effect on jets ${ }^{[9]}$, many of the details and the physical mechanisms involved are unclear. The recent experimental studies mentioned above have been directed to increasing understanding of the modification of the jet caused by different numbers and placements of tabs as well as the mechanism responsible for the effect of the tabs. While these experiments have shed some light on the phenomena of jet modification by tabs, further study of this effect is desirable.

To date there has been little use of numerical modeling in the study of the effect of tabs on jets. A significant exception is the very recent work of Grinstein, Gutmark, Parr, Hanson-Parr and Obeysekare ${ }^{[10]}$. They reported the results of a combined experimental and computational study of both reacting and non-reacting subsonic jets with circular cross-section. These jets were excited in an axisymmetric mode and tabs were present in the experiment and were modeled in the computation. The authors modeled the streamwise vortices generated by the tabs by including an azimuthally varying, steady radial velocity at the inflow boundary which was found to induce streamwise vortices downstream of the inflow. In all of their calculations five symmetrically placed tabs were modeled and they found good qualitative agreement with experiment.

In the present study numerical simulations are also used to evaluate the mixing effectiveness of tab-like devices located at the trailing edge of a single slot nozzle with an injector. This configuration was selected because of the simple nozzle surface geometry and the existence of experimental data to guide the computational simulations. We used a different, somewhat more direct method of modeling the streamwise vortices produced by the tabs than the method of Grinstein, et al. ${ }^{[10]}$. We also modeled the flow of several different numbers and configurations of tabs. The purpose of this study is three-fold. The first involves determining mixing rates for the undisturbed shear layer over a range of convective Mach numbers. The second involves determination of the placement and number of tabs at the nozzle trailing edge in order to achieve 
increased mixing and the determination of the influence of ejector duct sidewall boundaries. Finally, the third objective is to elucidate the physical mechanisms responsible for the increased spreading rate in the presence of tabs.

The calculations were based on the compressible three-dimensional Navier-Stokes equations. In this work the tab induced vortex strength and size were specified as input parameters for mixing rate optimization. Numerical simulations and comparisons to experimental data are performed at the specific flow parameters of the experiment. Calculations are also made over a range of shear layer convective Mach numbers to evaluate mixing effects due to shear layer temperature, density and velocity ratios. All of the results are compared on the basis of a mixing effectiveness parameter defined in the text.

The problem formulation, including equations, boundary conditions, the modeling of the tabs and the diagnostic parameters for the mixing are given in Section 2. Section 3 contains the results of the simulations including those on the sensitivity to grid sizes. A discussion of the basic physical mechanism responsible for the enhanced spreading of the jet is in Section 4 . Section 5 contains qualitative comparisons with the results of flow visualizations. Finally, a summary and conclusions are given in Section 6 .

\section{Problem Formulation}

The flow configuration is a jet exiting from a slot (of height D) into a square duct (of height and width $\mathrm{H}$ ) in which there is a coflow. The jet spans the duct and is located equidistantly from the top and bottom of the duct. Figure 1 is a schematic of the channel and coordinate system. In these calculations $H / D=4.38$ and the length of the channel is $23.76 \mathrm{D}=5.43 \mathrm{H}$. These particular dimensions were chosen so as to model the experiments discussed in Section 3.2. The jet speed is $U_{1}$ with temperature $T_{1}$, and the coflowing jet has a speed of $U_{2}$ and temperature $T_{2}$. In addition to the velocities and temperatures, the pressures or densities must also be prescribed at the inlet of the supersonic jet. We choose to set the pressures $P_{1}$ and $P_{2}$.

In addition to the slot width and the $\left\{U_{j}, T_{j}, P_{j}\right\}$ the flow is characterized by the Mach numbers of the jet and coflow at the inflow boundary

$$
M_{j}=\frac{U_{j}}{c_{j}}, \quad j=1,2,
$$

with the sound speeds

$$
c_{j}=\sqrt{\gamma R T_{j}}, \quad j=1,2,
$$

where $\gamma$ is the ratio of specific heats and $R$ the gas constant. In the calculations whose results are presented here, the jet was always supersonic and except for one case, the coflow was subsonic. 
It is now well known that the growth of the mixing layer between coflowing streams is dependent on, at least in part, the convective Mach number

$$
M_{C}=\frac{U_{1}-U_{2}}{c_{1}+c_{2}}
$$

The calculations reported here had a range of $M_{C}$ from 0.601 to 1.183 .

Finally, the flow is characterized by the jet Reynolds number. The appropriate length scale is the jet width $D$. The appropriate velocity scale is the difference between the inflow speeds of the jet and the coflow because it is the true measure of the shear in the mixing layer at the jet interface. Thus the jet Reynolds number is

$$
R e=\frac{\left(U_{1}-U_{2}\right) D}{\left(\mu_{1} / \rho_{1}\right)}
$$

with $\mu_{1}$ the viscosity and $\rho_{1}$ the density of the gas at the jet inflow. Typical values of $R e$ for the cases reported here are in the range $1.0 \times 10^{5} \leq R e \leq 2.5 \times 10^{5}$.

\subsection{Equations}

The governing equations for this flow are the mass conservation equation

$$
\frac{\partial \rho}{\partial t}+\frac{\partial}{\partial x_{j}}\left(\rho u_{j}\right)=0
$$

the compressible Navier-Stokes equations

$$
\frac{\partial\left(\rho u_{j}\right)}{\partial t}+\frac{\partial}{\partial x_{k}}\left(\rho u_{j} u_{k}\right)+\frac{\partial p}{\partial x_{j}}=\frac{\partial \sigma_{j k}}{\partial x_{k}}
$$

with

$$
\begin{gathered}
\sigma_{j k}=2 \mu\left(S_{j k}-\frac{1}{3} \delta_{j k} S_{l l}\right), \\
S_{j k}=\frac{1}{2}\left(\frac{\partial u_{j}}{\partial x_{k}}+\frac{\partial u_{k}}{\partial x_{j}}\right),
\end{gathered}
$$

and the energy equation

$$
\frac{\partial}{\partial t}\left[\rho\left(e+\frac{1}{2} u_{j} u_{j}\right)\right]+\frac{\partial}{\partial x_{k}}\left[\rho u_{k}\left(e+\frac{1}{2} u_{j} u_{j}+\frac{p}{\rho}\right)\right]=\frac{\partial}{\partial x_{k}}\left(u_{j} \sigma_{j k}\right)+\frac{\partial}{\partial x_{k}}\left(\kappa \frac{\partial T}{\partial x_{k}}\right),
$$

together with the equation of state,

$$
p=R \rho T \text {. }
$$

As usual, $\rho$ is the density, the $\left\{u_{j}\right\}$ are the velocity components, $p$ is the pressure, $e=C_{v} T$ is the internal energy per unit mass, $T$ is the temperature, $\kappa$ is the thermal conductivity, $C_{v}$ is the 
specific heat at constant volume and $\mu$ is the viscosity coefficient. The viscosity is computed using Sutherland's law

$$
\mu=\mu_{o} T^{3 / 2} /(110.0+T) .
$$

It is assumed that the bulk viscosity coefficient is zero and that $C_{v}$ and $C_{p}$, the specific heat at constant pressure, are constant. Finally, the Prandtl number is assumed constant and equal to 0.72 .

We also assume the flow to be laminar. In the experiments of Samimy, et al. ${ }^{[5]}$ and Zaman, et al. ${ }^{[8]}$ some of the configurations were, in their words, "nominally laminar" and others "nominally turbulent". In both cases they found that the experimental results were virtually identical. It appears that the dominant physical mechanism is the presence of the strong streamwise vortices generated by the tabs. Thus we expect that the laminar flow modeling in our calculation will not change the overall structure of the results.

The equations are approximated by finite differences on a grid with $\left(N_{x}, N_{y}, N_{z}\right)$ points in the $(x, y, z)$ directions, respectively. A uniform grid is used in the $x$ direction and a slightly stretched grid is used in the $y-z$ plane to take into account the boundary layers at the walls. Figure 2 shows a $(101 \times 101)$ grid in the $y-z$ plane. The slightly increased resolution near the sidewall boundaries is apparent as is the nearly uniform grid throughout the central portion of the domain. The finite difference equations are solved by a time accurate MacCormack predictorcorrector scheme (Peyret and Taylor ${ }^{[11]}$ ). The results of grid refinement studies are presented in Section 3.1.

\subsection{Boundary and Initial Conditions}

The inflow conditions are set as specified above. In most cases the top, bottom and side walls are taken to be impermeable, no-slip and insulated boundaries. In a few cases periodic boundary conditions are used in place of the side wall boundaries. This is done in order to asses the effect of the solid side walls on mixing for both cases with and without tabs. On the top and bottom walls $(z / H=0,1)$ the velocity is set to zero and a zero gradient of pressure and temperature is imposed. The sidewalls $(y / H=0,1)$, are treated the same as the top and bottom walls if they are taken to be solid. If the domain is taken to be periodic in $y$, the velocity, pressure, temperature and density at $y / H=0$ are equal to the velocity, pressure, temperature and density at $y / H=1$.

On the outflow boundary the velocity, temperature and pressure are obtained by extrapolation from the grid point upstream. This extrapolation is exact for quantities within the supersonic jet but is not so in the subsonic region outside the jet where its use can induce partial reflections. 
The effect of this outflow boundary condition, in particular its upstream effect, is discussed in detail in Section 3.1 where we show that the effect does not change our results in any significant way.

The initial condition is to set the velocity, temperature and pressure everywhere within the domain equal to the inflow velocity and then time step until a steady state flow field is reached. Generally, it is found that a few thousand time steps are required. As a criteria for convergence, the mass flow rate is computed and is found to vary by no more than $1 \%$ at any time during the simulations.

\subsection{Modeling the Tabs}

Possible physical mechanisms by which tabs produce streamwise vortices has been discussed by Samimy, Zaman and Reeder ${ }^{[5]}$; Zaman ${ }^{[6]}$; and Zaman, Reeder and Samimy ${ }^{[8]}$. One possible mechanism is the stripping and rolling up of the boundary layer by the tab. Another possible mechanism is the effect of an upstream pressure gradient caused by the presence of the tab. Either mechanism could produce streamwise vortices. For either mechanism, the direction of rotation of the vortex pair could be controlled by the orientation of the tabs ${ }^{[5]}$. Based on their experimental observations, Samimy, et al. ${ }^{[5]}$ conjectured that a delta or triangular shaped tab placed on edge of a jet with the apex leaning downstream would produce a pair of vortices of the "trailing vortex" type and if the apex were pointing upstream the pair of vortices would be of the "necklace vortex" type. In their terminology, the difference between these types is the sense of rotation. The sense of rotation of the "necklace vortex" pair is such that, between the vortex pair fluid is moved from the jet into the coflow while for the "trailing vortex" type, between the vortex pair fluid is moved from the coflow into the jet. In a later study, Zaman, et al. ${ }^{[8]}$ were able to show, via flow visualization, that tabs produced different flows depending on whether the apex of the delta tab pointed upstream or downstream. By way of comparison we note that Grinstein, et al. ${ }^{[10]}$ use the term "delta-wing vortex" instead of "trailing vortex" and "mushroom vortex" for "necklace vortex". In this paper we adopt the terminology of Samimy, et al. [5] and denote the trailing vortices as either "necklace" or "trailing".

In the present study it is not possible to directly add small physical tabs to the geometry because of the limitations on the spatial resolution in the code. Instead we model their effect by assuming that each tab generates a pair of counter rotating vortices. We introduce a simple model flow generated by these vortices on the inflow boundary. Figure 3 is a sketch illustrating the basic geometry. The point $\left(Y_{o}, Z_{o}\right)$ is the center of one of the vortices. The point $\left(Y_{j}, Z_{k}\right)$ is any grid point on the inflow boundary. The radial distance between these points is $r$. We assume that 
the vortex at $\left(Y_{o}, Z_{o}\right)$ generates a circumferential velocity, $V_{\theta}$, at $\left(Y_{j}, Z_{k}\right)$ which is given by

$$
V_{\theta}=V_{0}\left(\frac{b}{r}\right)\left[1-e^{-(r / b)^{2}}\right]
$$

with $b$ the scale of the vortex and $V_{0}$ the amplitude. Then at $\left(Y_{j}, Z_{k}\right)$ the cartesian components of the velocity are

$$
V_{j, k}=V_{\theta} \cos \theta \quad, \quad W_{j, k}=V_{\theta} \sin \theta .
$$

In a counter rotating vortex pair, one has a positive $V_{0}$ and the other has a negative $V_{0}$. At each grid point on the inflow boundary we sum up the contributions to $\left(V_{j, k}, W_{j, k}\right)$ from each of the vortices, yielding the total inflow values of $\left(V_{j, k}, W_{j, k}\right)$. In a similar way we modify the inflow pressure field to account for the vortices. The sense of rotation of the vortex pair is set by the sign of $V_{0}$. As will be shown below, if $V_{0}$ is positive the vortex pair is a "necklace" vortex pair and is a "trailing" vortex if $V_{0}$ is negative.

Because of the relative simplicity of the tab modeling, one can only expect that the major features of the actual flow field resulting from the presence of the physical tabs will be captured in the calculation. The fine details of the physical flow in the near field of the tabs will be missing from the calculations. Nevertheless, we expect to predict correctly the major features of the flow, the trends as the flow parameters are varied, and to be able to elucidate the basic physics of the tab-jet interaction.

\subsection{Measures of the Mixing}

A qualitative judgement of the effectiveness of tabs in promoting mixing between the jet and the coflow can be obtained by comparing the velocity fields of the same flow without tabs and with tabs. In addition to comparing the velocity fields, two measures of the effectiveness of the tabs were used.

The first measure of the mixing effectiveness is the increase in the jet thickness with tabs present as compared to the thickness with no tabs. The jet thickness, $\delta$, is defined by

$$
\delta(x)=z_{\text {upper }}(x)-z_{\text {lower }}(x),
$$

where $z_{\text {upper }}(x)$ is the position of the upper edge of the jet, averaged over the channel width and $z_{\text {lower }}(x)$ is the position of the lower edge of the jet, also averaged over the channel width. The definition of the edge of the jet is the $z$ position at which $U$ is equal to 1.02 times the speed of the main portion of the coflow.

The second measure of the mixing effectiveness is a mixing parameter, $\eta$. This is defined by

$$
\eta=1-\left(\frac{I_{1}}{I_{2}}\right),
$$


where

$$
I_{1}=\iint \rho u\left|T_{o x}-T_{o m}\right| d y d z
$$

and

$$
I_{2}=\iint \rho u\left|T_{o e}-T_{o m}\right| d y d z
$$

with the integration being over the cross section of the channel. In this definition, $T_{o x}$ is the local stagnation temperature, $T_{o e}$ is the stagnation temperature at the entrance to the channel and $T_{o m}$ is stagnation temperature of the fully mixed flow. The stagnation temperature of the fully mixed flow is given by

$$
T_{\text {om }}=\frac{\mathcal{M}_{1} T_{1}+\mathcal{M}_{2} T_{2}}{\mathcal{M}_{1}+\mathcal{M}_{2}}
$$

and the $\mathcal{M}_{j}$ 's are the mass flows of the jet (1) and the coflow (2); see Figure 1. A totally unmixed flow will have $\eta=0$ and a completely mixed flow will have $\eta=1$.

\section{Results}

We present, in the first subsection below, results showing the effect of varying the size of the computational grid. In the next subsection results for the case of no tabs are given. Some of these results are compared to existing experimental data. Others show the effect of periodic sidewalls as compared to solid sidewalls as well as the effect on the mixing of varying the convective Mach number. Finally, the results of simulations with tabs are presented in the last subsection.

\subsection{Grid Effects}

A series of grid refinement calculations were carried out in order to asses the grid effect on the accuracy of the results. The specific flow parameters are those of Case 1 in Table 1 . The conditions $\left(M_{1}=1.566, M_{2}=0.634, M_{C}=0.601\right.$ and $\left.R e=1.30 \times 10^{5}\right)$ are representative of the mid-range of the flow parameters used in the set of simulations. In these calculations $N_{x}$ was held constant at a value of 145 . The $\left(N_{y}, N_{z}\right)$ grids on the $y-z$ plane were taken to be $(51 \times 51),(76 \times 76),(101 \times 101)$ and $(126 \times 126)$.

Profiles of $U$, the streamwise component of the velocity, and the temperature, $T$, at $x / D=6.0$ are shown in Figure 4. These profiles are at $y / H=0.5$. The results for all four of the grids are shown in the plots. The thin viscous boundary layers on the top and bottom walls, the shear layers on the edge of the jet and the thermal boundary layers on the top and bottom walls can

be seen. It is clear that the results are somewhat different, depending on the grid. It can be seen that the coarsest grid results differ the most from the finest grid results. However, it is difficult 
to determine the magnitudes of the differences from this figure. For this reason we calculated the differences between the values of $U$ and $T$ on the $(51 \times 51),(76 \times 76)$ and $(101 \times 101)$ grids and the values on the $(126 \times 126)$ grid. Of course this can only be done at grid points common to all grids. These results, for $x / D=1.0,4.0,6.0$ and 10.0 are shown in Figures 5 and 6 . It is apparent that the differences between the results with $(51 \times 51)$ or $(76 \times 76)$ grids and the $(126 \times 126)$ grid are fairly large. The differences between the $(101 \times 101)$ grid and the finer grid are always less than or about equal to $5 \%$ and are usually about $2 \%$ or less. We concluded that using a $(101 \times 101)$ grid in the $y-z$ plane gives reasonable, within a few percent, accuracy. This conclusion is borne out by the results shown in Figure 7; a plot of $\eta$ as a function of $x / D$ for all four grids. For all of these cases shown $\eta$ increases rapidly up to about $x / D=2$ and then increases linearly, with a small slope, with increasing $x / D$. In all of these cases the slope is about the same; the difference occurs in the first two or three jet widths downstream of the entrance. The coarsest grid gives the largest values of $\eta$ and conversely. It is also clear that the results on the finest two grids are quite close and that we can extrapolate the values of $\eta$ to zero grid spacing.

As noted above, the velocity, temperature and pressure on the outflow boundary are obtained by extrapolation from the grid point upstream. This extrapolation is exact for quantities within the supersonic jet but is not so in the subsonic region outside the jet where its use can induce partial reflections. In order to assess the magnitude of these we carried out additional calculations. We noted that this treatment of the outflow caused what appears to be a small distortion of the flow over a distance of less than one channel height upstream of the outflow boundary. In the first of these calculations we verified that this distortion is due to the subsonic portion of the flow by setting both the coflow and the jet to be supersonic. The distortion disappears except for a small remnant in the subsonic viscous boundary layers on the walls. In the second calculation we sought a quantitative measure of how far the distortion extends upstream when the coflow is subsonic. In this calculation the grid in the $y-z$ plane was kept at $(101 \times 101)$ and the grid spacing in the streamwise $(x)$ direction was also held fixed but the number of grid points in the $x$ direction was reduced from 145 to 109 . Thus the streamwise extent of the computational domain was reduced by $25 \%$; this is called the short domain in contrast to the full domain with 145 grid points in the streamwise direction. We ran the calculation for the short domain using the flow parameters of Case 1 . If there were no effect of the outflow boundary conditions the flow field in the short domain would be identical to that in the first $75 \%$ of the full domain. Any deviations are due to the outflow boundary conditions and one can readily determine how far upstream from the outflow boundary a significant effect exists.

As was expected, the difference in flow quantities between the short and full domain at the same distance from inflow are largest within the wall boundary layer and are smallest in the supersonic jet. At a distance of $0.4 H$ upstream of the outflow of the short domain the differences in the velocity, temperature and pressure between the full and short domain are at most $3 \%$ 
in the wall boundary layer, $1 \%$ in the subsonic coflow and about $0.3 \%$ in the supersonic jet. Further upstream, at $1.06 \mathrm{H}$ from the outflow of the short domain, the differences in the velocity, temperature and pressure between the full and short domain are about $2 \%$ in the wall boundary layer, about $0.5 \%$ in the subsonic coflow and less than or equal to $0.1 \%$ in the supersonic jet. Further upstream these difference are even smaller. As will be seen from the results presented below, the wall boundary layers play no role in the interaction of the streamwise vortices with the jet. It is clear that the outflow boundary condition has virtually no effect on the flow upstream of about one channel width of the end of the computational domain. In general we do not use any of the computational results within a distance of one channel height upstream of the outflow boundary. Throughout the remainder of this paper, all results presented were obtained on a $(145 \times 101 \times 101)$ grid .

\subsection{Comparison with an Experiment}

A limited set of experimental measurements are available for the case of the mixing of a hot supersonic jet with a colder subsonic coflow. The fluid of both the jet and the coflow was air. The source of the coflow was air at atmospheric pressure and temperature. The experimental domain was $0.16 \mathrm{~m}$ long ( $x$ direction) with a cross section of $0.0442 \mathrm{~m}(y) \times 0.0442 \mathrm{~m}(z)$. The thickness of the jet at the inflow was $0.0101 \mathrm{~m}$. Thus we set $D=0.0101 \mathrm{~m}$ and $H=0.0442 \mathrm{~m}$ for the computation. For the simulation we set the temperature and pressure of the coflowing air to be $T_{2}=300^{\circ} \mathrm{K}$ and $P_{2}=1.01325$ bar, respectively. The pressure and temperature in the pressure vessel driving the jet are known. The hot, high pressure air flows out of the pressure vessel through a nozzle but we had no information on the geometry of the nozzle. The jet expands and cools while flowing through the nozzle.

Time averaged measurements of $U$, the $x$ component of the velocity, were made at a number of stations on the centerline of the jet beginning just outside the nozzle. In addition, profiles of $U$ as a function of $\mathrm{z}$ were made at three locations downstream from the exit plane of the nozzle. These measured data are shown in Figures 8 (as a $\bullet$ ) and 9 (as a + ). Some of this experimental data is used to obtain the inflow conditions for the simulation.

First, the profile data (Figure 9) show that the coflow speed was $U_{2}=220 \mathrm{~m} / \mathrm{s}$. The measured values of the velocity on the jet centerline are shown in Figure 8a (denoted by $\bullet$ ). The measured velocity drops as the jet exits from the nozzle, then increases further downstream and continues to oscillate with distance until it begins to decrease monotonically at about $x / D=13.0$. The decrease in the jet speed after exiting from the nozzle indicates that the jet pressure is increasing. That is, the jet was over expanded in the nozzle and undergoes a compression just outside the nozzle. This is followed by a series of subsequent expansions and compressions, with decreasing amplitude, further downstream. This data shows that we must set the jet pressure slightly 
less than that of the coflow (which is at atmospheric pressure). We adjusted the pressure, temperature and speed of the jet in order to get reasonable agreement with these centerline velocity measurements. Thus for the jet we set $P_{1}=0.92 \times P_{2}, T_{1}=490^{\circ} \mathrm{K}$ and $U_{1}=695.0$ $\mathrm{m} / \mathrm{s}$. With these values at the inflow, the Mach number of the coflow is 0.634 and that of the jet is 1.566 . The Reynolds number of the jet is $1.20 \times 10^{5}$.

Figure 8 a shows plots of the computed $U$ on the jet centerline (solid line) and the measured values $(\bullet)$. There is fair agreement; it appears that there is a small offset between the location of the peaks of the computed $U$ and the measured values. Shifting the computed curve by about $0.4 \mathrm{D}$ would bring it into good agreement with the measurements. The oscillations in $U$ are caused by a sequence of compression and expansion standing waves in the channel outside the nozzle. These pressure oscillations are obvious in the computed centerline pressure distribution shown in Figure 8b. The maxima and minima in the calculated velocity and pressure are exactly out of phase. The presence of the expansion and compression waves means that the jet is undergoing

small spatial oscillations in size on top of a general spreading of the jet. The calculated profiles of $U$ are compared to the measured profiles in Figures 9 at $x / D=1.0,2.5$ and 10.0. In each of these figures the solid line is the calculated value and the + denote the measured values. There is, again, fair agreement between the calculated and measured profiles. The differences between calculated and measured values of the maximum speed is mainly due to the shift in the maximum value on the centerline seen in $8 \mathrm{a}$. The measurements also show a somewhat thicker jet than do the calculations. We attribute this to the fact that the calculations are for a laminar flow while the mixing layer between the jet and the coflow was turbulent in the experiments. The agreement between calculation and experiment is good enough to suggest that the major features of the flow, the trends as the flow parameters are varied and the basic physics of the tab-jet interaction can be captured by the calculations.

\subsection{Mixing: No Tabs}

In this section we first show how the mixing varies with flow parameters in the absence of tabs. Figure 10 is a plot of the variation of the mixing parameter $\eta$ with downstream distance. The results shown in this figure illustrate how the mixing changes with changes in the convective Mach number. The curves are labeled with the value of $M_{C}$. There are two curves for $M_{C}=0.601$. The solid one is the result of using the input data of Case 1 in Table 1 and the dashed curve uses the input data of Case 2. The only difference is that the jet pressure for Case 2 is $92 \%$ of that for Case 1, and is clear that it has no effect on the mixing.

In Figure 10 results are shown for three different values of $M_{C}, 0.601$ (Cases 1 and 2)), 0.782 (Case 5) and 1.183 (Case 4). With $M_{C}=1.183$ (Case 4), $\eta$ is very much larger than with $M_{C}=0.782$ or 0.601 at distances more than one jet thickness downstream of the entrance. As 
compared to the Cases 1,2 and 5, the jet speed is much higher for this Case although the jet temperature is the same. Again as compared to Cases 1,2 and 5, the coflow speed is much lower and its temperature is slightly lower. The net effect is that the Mach number of the jet is increased to 2.141, that of the coflow is reduced to 0.158 and the jet Reynolds number increases to $2.473 \times 10^{5}$. The overall increase in $\eta$ due to the increase in $M_{C}$ is substantial; at $x / D=10.0$, $\eta$ with $M_{C}=1.183$ is 2.3 times larger than with $M_{C}=0.601$ and 1.6 times larger than with $M_{C}=0.782$. The same trend is also observed when the variation of $\delta / D$ with $x$ is compared at different values of $M_{C}$ as in Figure 11. Again we see that changing the inflow pressure has almost no effect on the mixing measure but changing $M_{C}$ does. Changing $M_{C}$ does not have as large an effect on $\delta$ as on $\eta$; the magnitude of the change in $\delta / D$ is, at $X / D=10.0$, less than $20 \%$.

It is obvious that changing $M_{C}$ has a large effect on $\eta$. In order to gain insight into the reason for this, we show in Figure 12 profiles of the normalized streamwise velocity component, $U / U_{o}$, and temperature, $T / T_{o}$, as a function of the dimensionless height, $z / H$, at $y / H=0.5$ and $x / D=4.0$ at different values of $M_{C}$. In each case the velocity and temperature are scaled by the values of the velocity $\left(U_{0}\right)$ and temperature $\left(T_{o}\right)$ of the jet at the inflow boundary. Note that in this figure the solid line denotes results for $M_{C}=0.601$; the long dash line for $M_{C}=0.782$; and the short dash line for $M_{C}=1.183$. In order to increase $M_{C}$ we decrease the speed of the coflow relative to the speed of the jet. This is apparent in the $U / U_{0}$ plot. The increased shear led to a progressive thickening of the velocity mixing layers between the jet and the coflow. At the highest value of $M_{C}(1.183)$, the increased viscous dissipation leads to a local increase in the temperature within the mixing layers; this can be seen in the $T / T_{0}$ plot. As a result of these, apparently, small modifications of the flow there was a significant increase in the value of $\eta$ just downstream of the entrance.

The results shown here are for a purely laminar flow; the mixing is controlled by laminar diffusion of momentum and heat. From the curves of Figures 10 and 11, it is apparent that the jets mix rapidly over a distance of three to four jet widths downstream from the inflow boundary. Thereafter the rate of growth of the jets is quite modest. In this region the increase of $\eta$ with distance is about the same for each of these cases. This can be seen by noting that the curves are approximately parallel after about four jet widths downstream. The initial rapid thickening of the mixing layer is primarily due to the step increases in velocity and temperature between the jet and the coflow, imposed at the inflow boundary, and their subsequent spread by laminar mixing.

It may seem surprising that, for a purely laminar flow, the mixing parameter, $\eta$, increases with $M_{C}$. However it can be shown that this also occurs in the case of the laminar, compressible mixing layer. To see this we take the mean profiles of $U$ and $T$ in the mixing layer to be the similarity solutions given by Grosch and Jackson ${ }^{[12]}$. In their notation the dimensionless speed 
and temperature in the coflow are scaled to be one and the dimensionless speed and temperature in the jet are scaled to be $\beta_{U}$ and $\beta_{T}$, respectively. With this notation the convective Mach number is

$$
M_{C}=\left(\frac{\beta_{U}-1}{\beta_{T}+1}\right) M
$$

with $M$ the the Mach number of the coflow. Holding $M$ fixed, $M_{C}$ increases with increasing $\beta_{U}$. Substituting the similarity solutions ${ }^{[12]}$ into the definition of $\eta$ (equations $15-17$, above) it is found that $\eta$ increases with increasing $\beta_{U}$, i.e. with increasing $M_{C}$. This effect occurs in the laminar mixing layer (which can serve as an approximation for the mixing layers on the edges of the jet) in the absence of instability waves and large scale turbulent structures. If there were instability waves in these numerical calculations for the jet their growth rate would

decrease with increasing $M_{C}{ }^{[13]}$. The shear layers between the jet and the coflow are inviscidly unstable. Because the convective Mach number is greater than or equal to 0.6 in the calculation, the growth rates of the instabilities are much smaller than at zero Mach number. The grid resolution is sufficient to represent the mean flow profiles in these shear layers, however it is not suffuicient to properly represent the structure of the instability waves. In our calculations we never observed any instability waves. We note that the experimental results presented in the papers of Samimy, et al. and Zaman, et al. also show no evidence of instability waves. It appears that the strong streamwise vorticity induced by tabs dominates the flow dynamics and that instabilities are relatively unimportant. Again if there were large scale turbulent structures present in these calculations we expect that their effect on the spreading would decrease with increasing convective Mach number ${ }^{[14]}$. Because there are no instability waves or large scale turbulent structures in these calculations these effects with increasing $M_{C}$ are not seen.

\subsection{Mixing: Tabs}

In the calculations reported here we used zero to eight of these model tabs; that is, pairs of counter rotating vortices. For an even number of these model tabs half are on the upper edge of the jet inflow and half are on the lower edge. If the number of tabs is odd, the additional tab is set on the upper edge. On both edges the tabs are uniformly spaced; for example for the case of six tabs they are centered one quarter, half and three quarters of the way across the span. In some cases we choose $V_{0}$ to be positive, hence an individual pair of vortices is of the "necklace" type. This has the effect that the induced flow at the centerline of the jet and near the sidewalls is away from the sidewalls. In other cases we choose $V_{0}$ to be negative, producing "trailing" vortices and an induced flow at the centerline of the jet and near the sidewalls toward the sidewalls. As will be discussed in the next section the results of the calculations show that the magnitude of the mixing depends on which type of vortex is generated. 
Figure 13 is a plot of the mixing effectiveness parameter, $\eta$, as a function of $x / D$ for different numbers of tabs. The curves are labeled with the total number of tabs. Those curves additionally labeled with " $T$ " are the result of calculations with negative $V_{0}$ and each pair of vortices is of the "trailing" type; the other curves were the result of calculations with positive $V_{0}$ and each pair of vortices is of the "necklace" type. The calculations with tabs use the same inflow data as that without tabs. In all cases one can see that in the near field of the entrance, say within two or three $D$ downstream of the inflow boundary, the presence of the tabs has only a slight effect on $\eta$. However, beyond about three jet thicknesses downstream, the mixing is greatly increased by the presence of the tabs. Throughout this region of the computational domain $\eta$ is increasing linearly with $x / D$. It is obvious that the slope of the $\eta-x / D$ curve depends on the number of tabs. The biggest increase in the slope of this curve occurs when a few tabs are added. Adding more tabs then has a diminishing effect. For example, at $x / D=14.0$, the value of $\eta$ with two tabs is 1.56 times as large compared to no tabs, with four tabs the ratio is 2.08 , with six tabs it is 2.44 and going to eight tabs only increases this ratio to 2.49 . The mixing parameter increases by $56 \%$ when going from zero to two tabs; by $33 \%$ going from two to four tabs; by $17 \%$ going from four to six tabs and only by $2 \%$ going from six to eight tabs. Because of the near linearity of these curves beyond $x / D \approx 3.0$, this holds at all $x / D$. The basic reason for this decrease in the marginal gain in mixing is that adding more tabs in a channel of fixed width causes them to be closer together. This increases the mutual interference and decreases the mixing.

The curves labeled $2-T$ and $4-T$ are the result of a computation with two and four tabs, respectively, but with $V_{0}$ negative and, in contrast to the other computations, vortices of the "trailing" type. For these two cases the vortex induced flow on the jet centerline near the sidewalls is directed towards the sidewalls. The mixing for both these cases is substantially less than for the corresponding cases with two and four tabs having positive $V_{0}$ and "trailing" vortices. The flow fields for these cases are discussed and compared in the next section.

To determine the effect of the sidewalls on mixing a few calculations a re carried out with periodic boundary conditions in the $y$ direction. A total of six tabs were used. They were positioned three on each of the upper and lower edge of the jet. The centers of the pairs of counter rotating vortices modeling the tabs were placed at $y / H=1 / 6,1 / 2$ and $5 / 6$. This modeled an infinite set of tabs with a spacing of $1 / 3$ on centers. The plot of $\eta$ vs $x / D$ is shown in Figure 13 by the short dashed line. For small values of $x / D, \eta$ for the periodic flow is small; however as $x / D$ increases $\eta$ increase faster than for the cases with solid side walls. Towards the end of the computational domain the periodic case has the largest values of $\eta$. We think that this is due to the decrease in the dissipation because of the removal of the side boundaries. The flow field for the periodic case will be discussed in more detail below.

The effect of tabs on the jet thickness is shown in Figure 14 where we plot $\delta / D$ as a function of $x / D$. As with $\eta$, the presence of tabs has the effect of increasing $\delta$ but, relatively, not as much. 
Note that the periodic case has the largest values of $\delta$ at small $x / D$ and is nearly identical to the solid side wall case with six tabs at larger $x / D$. The case of eight tabs yields nearly the same thickening of the jet as does that with only four tabs. This, again, shows the decreasing effectiveness of having more than six tabs. Overall, beyond $x / D \approx 10.0$ there is a maximum increase in $\delta / D$ of $25 \%$ with six tabs and solid side boundaries or periodic side boundaries as compared to the no tab case. All of these curves show oscillations in $\delta / D$ with $x / D$. The value of $\delta$ is quite sensitive to small perturbations in the shear layers separating the jet and the coflow. The presence of the tabs caused small oscillations in the pressure field near the inflow boundary. These propagate downstream and generate a small thickening and thinning of the jet; observed as the oscillations in $\delta / D$. The flow field generated by the vortices which cause this greatly enhanced mixing will be discussed in detail in a later section.

\section{Flow Structure}

These results show that tabs cause increased mixing of the hot compressible jet. In this section we present results showing the flow structure in order to elucidate the mechanism which causes this. We show the flow structure for both positive and negative $V_{0}$ with associated vortices of the "necklace" type and of the "trailing type, respectively. Only the velocity field is shown in the figures which are discussed below. The other dependent variables have a similar structure. All of the results shown here are done with inflow conditions of Case 2 as given in Table 1.

We first show the velocity field in the absence of tabs. Figure 15 shows contours of the streamwise component of the velocity, $U$, at $x / D=14.0$. The central, high speed jet has a uniform core (speed of $631.165 \mathrm{~m} / \mathrm{s}$ ) extending over the central $20 \%$ of the channel. Away from the sidewalls, the jet shear layers are uniform across the span and each has a thickness of about three quarters of the size of the core. The coflow jets above and below the central jet also have a uniform core. The wall boundary layers are clearly visible. There is some slight distortion of the side wall boundary layers. In these regions modest values of $(V, W)$ are found due to the growth of the boundary layers with downstream distance. Except for the scales, i.e. the speed of the jet core, the thickness of the mixing layer between the jet and the coflow, and so forth, the flow at all sections is the same. There is a laminar mixing layer which is growing slowly with downstream distance. Both the hot, high speed jet and the coflow maintain their existence with no global

mixing. Note that even fourteen jet widths downstream of the inflow there is no indication of any instabilities in the mixing layers between the jet and the coflow.

We next show the flow field for the same inflow values, but with six tabs and positive $V_{0}$. Each of the vortex pairs generated by the tabs are of the "necklace" type. At the inflow of the domain there are three tabs on the upper edge of the jet and three on the lower edge. These are placed 
at $y / H=1 / 4,1 / 2$ and $3 / 4$. Contour plots of $U$, the streamwise velocity component, and vector plots of the transverse components, $(V, W)$ are shown at a number of streamwise locations. These illustrate the detail of the downstream evolution of the combined jet-vortex flow field.

Figure 16 is a plot of the vector field of $(V, W)$ in the $y-z$ plane of the channel at the channel entrance $(x / D=0.0)$. The set of twelve counter rotating vortices that model the six tabs are obvious in this figure and it is clear that each pair is of the "necklace" type. The inner vortices are slightly weaker than the outermost ones because of the mutual interference of those on either side. The outermost vortices are only interacting with vortices on the inside. The maximum value of the $(V, W)$ velocity in this plane is $79.0 \mathrm{~m} / \mathrm{s}$.

A similar plot at $x / D=1.0$ is shown in Figure 17. Some small distortion of the flow, as compared to $x / D=0.0$, can be seen. All of the vortices have moved slightly away from the centerline, $z / H=0.5$, with the outermost vortices a bit above/below the inner ones. The maximum value of the $(V, W)$ velocity in this plane is $85.0 \mathrm{~m} / \mathrm{s}$, slightly higher than at inflow. Figure 18 is a contour plot of the streamwise velocity component, $U$, on the same plane. The cross stream waves in the mixing layers between the jet and the coflow are the direct result of the streamwise vortices. Comparing Figures 17 and 18, the peaks in Figure 17 coincide with the outflows and the troughs with the inflows from the jet core in the $(y-z)$ plane. These are directly due to the six pairs of vortices generated by the tabs. Note that the distortion of the mixing layer is symmetric about its undisturbed position at this downstream location.

Figure 19 is a plot $y-z$ plane at $x / D=4.0$ of the vector field of $(V, W)$. Note that the four interior vortices have weakened considerably. The innermost ones still exist, albeit they are very weak. The next outermost ones have virtually disappeared. The outermost sets of vortices have moved slightly towards the centerline, in contrast to the situation at $x / D=1.0$. The maximum value of the $(V, W)$ velocity in this plane is $105.4 \mathrm{~m} / \mathrm{s}$, higher than at $x / D=1.0$. Using this figure alone it is difficult to identify any of the organized vortex pairs generated by the tabs.

The further downstream evolution of the flow is shown in Figures 20 and 21 . The $(V, W)$ vector field at $x / D=10.0$ is plotted in Figure 20 and Figure 21 is a contour plot of the streamwise velocity component, $U$, on the same plane. The maximum value of the $(V, W)$ velocity in this plane is $69.1 \mathrm{~m} / \mathrm{s}$, substantially lower than at $x / D=4.0$. The vortex structure in Figure 17 retains much of the symmetry observed at the upstream planes but also shows non-symmetric features, particularly in the central region of the flow. The four outermost vortices are much stronger than the inner ones and have moved off towards the corners of the channel. Again using only this figure it is difficult to identify the original set of vortex pairs. The velocity contours shown in Figure 21 are very symmetric and has evolved markedly from the field at $x / D=1.0$ shown in Figure 18. Fingers of the hot, high speed fluid from the jet have been moved into the colder, low speed coflow and in return fingers of the colder, low speed fluid have been transported 
into the central jet and have split the original jet into 3 distinct jets on the centerline. These contours show a very high degree of symmetry. It is clear that the interpenetrating fingers have been generated by the action of the streamwise vortices.

Figures 22 and 23 show the same flow field further downstream, at $x / D=14.0$. The further evolution of the flow from that at $x / D=10.0$ is clear. The outer vortices have moved further into the corners of the channel and are more asymmetrical. The inner vortices are much weaker and more disorganized than upstream and appear to be in the process of merging. The contours of $U$, the streamwise component of the velocity, as seen in Figure 23 are also somewhat distorted but retain much of the symmetry found upstream. The core jet has shrunk, compared to upstream, the high speed fingers are nearly impinging on the channel walls, the low speed fingers have nearly merged in the central part of the channel and the shear layers have thickened further. Finally, it should be noted that the most prominent features in Figure 23 are the hot, high speed fingers of fluid displaced outward from the jet into the coflow.

Similar evolution of the flow field was observed with different numbers of tabs with $V_{0}$ positive, thus generating "necklace" vortices. To show this, we give a number of results for the case of four and two tabs. Results are also given for negative $V_{0}$; these illustrate the effects of "trailing" vortices on the overall flow.

Figure 24 shows vectors of $(V, W)$ on the inflow boundary, at $x / D=0.0$ with positive $V_{0}$. There are two tabs on both the upper and lower edges of the jet at $y / H=1 / 3$ and $2 / 3$. The maximum value of the velocity on this plane is $84.5 \mathrm{~m} / \mathrm{s}$. This is slightly higher than was the case with six tabs $(79.0 \mathrm{~m} / \mathrm{s})$ and is due to the reduction in the mutual interference. Note that with positive $V_{0}$ and "necklace" type vortices, the flow along the centerline $(z / H=0.5)$ is away from the side boundaries in the region near the side walls. Figures 25 and 26 show the result of the downstream evolution of the flow field with four tabs and positive $V_{0}$. Figure 25 shows the vectors of $(V, W)$ on the plane at $x / D=14.0$; there is a maximum value of the velocity of $56.9 \mathrm{~m} / \mathrm{s}$. Figure 26 shows the corresponding contours of $U$. From Figure 25, we see that the inner vortices have paired, interacted strongly, have weakened considerably and nearly disappeared. However, the outer vortices have strengthened and moved towards the upper and lower boundaries. This field is not quite symmetric about the $y$ and $z$ centerlines. The similarity to the $(V, W)$ field, at the same $x / D$ location, resulting from six tabs (Figure 22) is clear. The $U$ contours in Figure 26 show a high degree of symmetry. The interleaving of fingers of hot, high speed fluid and cold, lower speed fluid is present with the fingers of hot, high speed fluid being the most prominent feature. Because of the interaction with the upper and lower walls, the fingers are beginning to curl over. In this case, the number of fingers is reduced to two on each side of the jet as compared to Figures 21 and 23 where three fingers are seen on each side of the jet. This is because there are four tabs instead of six. Again, the core of the high speed jet has nearly been split, into two distinct jets in this case as compared to three for the case of six tabs (see Figure 23). 
The results shown in the next three figures $(27-29)$ are also for the case of four tabs but $V_{0}$ has been replaced by $-V_{0}$, reversing the direction of rotation of the vortices. Thus the vortices are of the "trailing" type as compared to the "necklace" type. Figure 27 shows vectors of $(V, W)$ on the inflow boundary, $x / D=0.0$. As before, there are two tabs on both the upper and lower edges of the jet at $y / H=1 / 3$ and $2 / 3$. The maximum value of the velocity on this plane is 84.5 $\mathrm{m} / \mathrm{s}$ but the sense of rotation is reversed. Note that the sense of rotation of the vortices is such that the flow along the centerline $(z / H=0.5)$ is towards the side boundaries in the region near the side boundaries. Figure 28 shows the vectors of $(V, W)$ of this case at $x / D=14.0$. This is a flow field very different from that of Figure 25. The change in the sense of rotation of the vortices causes a different evolution. The inner vortices have paired and moved away from the centerline. They are generating a strong outflow from the jet in the region of $y / H=0.5$. As compared to their original positions, the outer vortices have only moved a little towards the side walls and there is virtually no displacement of them towards the top and bottom walls. The result on the $U$ field is shown in Figure 29 and it is substantially different from that resulting from the "necklace" vortices as seen in Figure 26. A large finger of hot, high speed fluid is ejected towards both the upper and lower walls of the channel from the jet along the $y / H=0.5$ centerline. This leaves a single, small high speed core in the center of the channel. The jet core has also been split into two other pieces which have been moved towards the sidewall boundaries by the outer set of vortices. This figure thus shows five different jets of the hot, high speed fluid of which the largest are the two in close proximity to the side walls. Also, note the high shear near these boundaries as the high speed jet core is advected towards the sidewalls.

An examination of the configuration of the vortices on the inflow plane suggests why arrays of "necklace" vortices should evolve downstream so differently from the evolution of "trailing" vortices. Consider the "necklace" vortex pair to the left of $y / H=0.5$ and above $z / H=0.5$ (the upper left quadrant) in Figure 24. The mutual induction of this pair is such that it has a tendency to move towards the top, $(z / H=1.0)$, of the channel. Each of the other vortex pairs, considered alone, would also tend to move away from the midplane of the channel and toward the top or bottom boundaries. Next consider the innermost sets of vortices. They are parts of different "necklace" vortices but they also have a mutual induction. This tends to move this inner set towards the $z / H$ midplane of the channel. Thus the total induction velocity of the innermost set is small because of cancellation. This is not true for the outer vortices because they are farther away from the members of the other pair. Thus the outer members of the array move toward the top/bottom boundaries. The in ner members are only slightly moved from their original position and, because of their opposite sense of rotation, they tend to cancel each other. The result is the far downstream configuration of the "necklace" vortices shown in Figure 25. These outer vortices cause the formation of the four fingers of hot, high speed fluid, two on each side of the channel midplane. Note that the fingers lie just on the inner side of the dominant vortices. With further downstream evolution the fingers tend to roll up on themselves. 
The "trailing" vortices seen in Figure 27 effect each other differently than the "necklace" vortices. The mutual induction of each pair tends to move them towards the centerline $(z / H=0.5)$ of the high speed jet. As with the "necklace" vortices, the innermost pair of vortices, although being members of different pairs of "trailing" vortices also interact mutually. This interaction tends to move each of them away from the centerline at $z / H=0.5$ and towards the upper and lower walls. Thus the inner vortices tend to pair, forming an inner "necklace" pair, and move toward the top and bottom of the channel. Again, they also tend to mutually cancel. The outer vortices basically stay in place and transport the high speed fluid of the jet core out of the center and towards the sidewall boundaries. This is seen in Figure 28; at $x / D=14.0$ the inner pairs have weakened and moved towards the upper and lower boundaries and the outer pair are only slightly modified.

The different interactions of "necklace" versus "trailing" vortices is also seen quite clearly when modeling a single tab on each edge of the jet. Figure 30 shows vectors of $(V, W)$ on the inflow boundary, at $x / D=0.0$ with one tab on each of the upper and lower edges of the jet at $y / H=$ $1 / 2$. Here $V_{0}$ is positive and the vortices are of the "necklace" type. The maximum value of the velocity on this plane is $88.4 \mathrm{~m} / \mathrm{s}$. The result of the streamwise evolution is shown in Figure 31 . The maximum value of $(V, W)$ on this plane is $64.6 \mathrm{~m} / \mathrm{s}$. Because of their mutual interaction the pair of vortices above the jet have moved towards the upper wall and that below towards the lower wall. Contours of $U$ for this case are shown in Figure 32. In this case, as might be expected, only a single finger of hot, high speed fluid is moved into the coflow on each side of the jet. The fingers show great symmetry about both the $y$ and $z$ centerlines. At this location, these fingers are just beginning to interact with the upper and lower boundaries. The mixing layer between the jet and the coflow is distorted but there is still a coherent high speed core of the jet spanning the channel.

Figure 33 shows vectors of $(V, W)$ on the inflow boundary, at $x / D=0.0$ again with one tab on each of the upper and lower edges of the jet at $y / H=1 / 2$. However, here $V_{0}$ is negative and the vortices are of the "trailing" type. The maximum value of the velocity on this plane is $88.4 \mathrm{~m} / \mathrm{s}$ just as for the "necklace" vortices. The mutual interaction of these "trailing" vortices tends to keep them close to the centerline of the channel. This is just what is seen in Figure 34 which is the result of their streamwise evolution. The maximum value of $(V, W)$ on this plane is $50.8 \mathrm{~m} / \mathrm{s}$ which is only about $79 \%$ of the maximum of the previous case. Both pairs of vortices have a slightly increased spanwise separation but have remained close to the centerline of the channel. Contours of $U$ for this case are shown in Figure 35. The vortex circulation has pinched the jet into a pair of jets of hot, high speed fluid, one on each side of the $y / H$ centerline of the jet. Again there is great symmetry about both the $y$ and $z$ centerlines. These jets are interacting with the side walls and the mixing layer between the jet and the coflow again shows substantial distortion. 
Finally, Figure 36 shows contours of $U$, at $x / D=14.0$, for the case of three tabs on both the upper and lower edges of the jet and with periodic boundary conditions in $y$. The tabs are placed at $y / H=1 / 6,1 / 2$ and $5 / 6$. Note that because periodic boundary conditions were imposed in $y$ the domain extends from $y / H=0.0$ to $y / H=1.04$. The maximum value of the $(V, W)$ velocity on the inflow plane was $84.6 \mathrm{~m} / \mathrm{s}$. The flow field is similar to that of Figure 23; that case also has six tabs. The major difference is the distortion introduced by the solid side walls as seen in Figure 23. In Figure 36, the periodic case, the magnitude of $U$ in the central jets and in the high speed fingers is somewhat greater while $U$ in the low speed fingers is somewhat smaller than in the corresponding case with solid side wall boundaries. It appears that this is due to the decrease in energy dissipation resulting from periodic rather than solid side walls.

The results shown in these figures reveal the basic physical mechanism of the interaction of the vortices generated by tabs with a hot jet. The vortices transport the hotter, higher momentum fluid from the central region of the jet to the lower momentum region of the coflow and vice versa. They thus act to increase the $z$-ward transport of $x$ momentum. This same process is, of course, also occurring by diffusion but the vortex transport is clearly more effective.

These results also show that the initial configuration of "necklace" type vortices on both the upper and lower edges of the jet is generally unstable. The innermost vortices interact strongly with each other, tend to be displaced, and are dissipated, starting with the next to the outermost and proceeding inward. The outermost vortices tend to become stronger and move toward the top and bottom of the channel. In contrast, configurations of "trailing" vortices tend to be much more stable. Inner pairs do interact and move towards the upper and lower boundaries but the outer ones tend to remain in place.

\section{Comparison with Experiments}

Finally, a qualitative comparison can be made between the results presented here and the results of experiments. In particular, Samimy, et al. ${ }^{[5]}$ and Zaman, et al. ${ }^{[8]}$ give flow visualizations of the jets with and without tabs. These visualizations are laser sheet illuminated cross sections of the jet. In these the brightest bands correspond to the mixing layer between the jet and the coflow. These experiments were done with jets of circular cross section with the boundaries far away from the jet. The simulations reported here were done with rectangular jets exiting into a channel with the side wall bounding the jet. Nevertheless, in spite of the different geometry, there is very good qualitative agreement between our results and theirs.

Samimy, et al. ${ }^{[5]}$ used tabs that appear to have generated "trailing" vortices. The flow visualiza- 
tions with two tabs on opposite ends of the nozzle diameter are shown in their Figures 7(Cont) on page 615 and Figures 8 through 11 on page 616 . These visualizations were obtained with different nozzles, tab size and shape and different Mach numbers. In all cases the basic structure of the disturbance produced by the tabs is the same; there is a "bulge" from the outer fluid into the mixing layer between the jet and the outer fluid and this "bulge" grows with downstream distance. In some of the visualizations (see their Figures 7 (Cont.) and 8, in particular) the jet bifurcates. The calculated contours of $U$ shown in Figure 35 of this paper show a very similar bifurcation of the plane jet at $x / D=14.0$ caused by two "trailing" vortex pairs, one on each side of the jet. This process begins near the entrance plane as inward "bulges" in both sides of the jets, again very similar to the visualizations shown by Samimy, et al. ${ }^{[5]}$.

Similar visualizations for four tabs are given by Samimy, et al. ${ }^{[5]}$ in their Figure 7 (Cont.). The originally circular mixing layer is strained into four fingers extending radially outward from the jet with indentations between the fingers. A similar pattern is seen in Figure 29 which contains contours of $U$ for the case of two sets of "trailing" vortices on each side of the jet. In between the pairs of vortices a finger of the high speed fluid from the jet extends into the coflow. A similar, but smaller, finger also appears between each vortex pair and the wall. Between these fingers indentations can be seen, aligned with the centers of the vortex pairs. The fingers are clearly the result of the outward flow induced by the interaction of sets of adjacent vortex pairs. Again, there is good qualitative agreement between the results of the simulations and the flow visualizations.

Similar flow visualizations were given by Zaman, et al. ${ }^{[8]}$ for jets of circular cross section with two, four and six tabs spaced uniformly around the jet. By changing the orientation of the tabs with respect to the the jet flow direction, they were able to produce both "trailing" and "necklace" type vortices and these generated different flow configurations. Zaman, et al.[8] showed visualizations at one $x / D$ for zero, one, two and four tabs with apexs leaning downstream and generating "trailing" vortices in their Figure 8. Their Figure 9 shows visualizations for two tabs generating "trailing" vortices at several streamwise locations. Both of these Figures show results very similar to the results of the simulations; "trailing" vortex pairs on opposite sides of the jet cause a bifurcation of the jet and two such sets of vortex pairs in close proximity generate a finger of the high speed fluid from the jet with indentations between the fingers. For the case of the tabs having their apex leaning upstream, they had predicted that the vortices would be of the "necklace" type and the flow would be different from the other cases. This was borne out by their results, for two tabs, shown in their Figure 13. The "necklace" vortices produced an outward "bulge" of the high speed jet flow. Very similar results are shown in Figure 32 here. This shows contours of $U$ for two sets of "necklace" vortices, one on each side of the jet. The outward "bulge" of the fluid from the jet with little distortion of the remainder of the jet is just as seen in the flow visualizations. 


\section{Summary and Conclusions}

The flow field consisting of a hot, compressible jet exiting into a slower moving, colder ambient flow was calculated using a compressible, three dimensional Navier-Stokes solver. Reasonable agreement was obtained with experimental results. We have shown that the effect of tabs at the edge of the jet can be modeled by replacing each tab with a pair of counter rotating vortices. Both "necklace" and "trailing" type vortices can be simulated, depending on the sense of rotation of the model vortices. Calculations of the flow with these present showed that the presence of the tabs increased the thickness of the jet about $25 \%$ compared to the flow without the tabs, depending on the assumed strength of the vortex circulation. The mixing parameter $\eta$ can be increased by a factor of about 2.5 by using six tabs. The effect of the sidewall boundaries on the mixing was also determined by repeating the calculation with six tabs with periodic boundary conditions in the cross stream direction. It was found that, with these boundary conditions, the mixing increased somewhat.

The results of the calculations elucidated the basic physical mechanism of the interaction of the vortices generated by tabs with a hot jet and that of the increased jet thickening and increased mixing. The streamwise vortices transport the hot, higher momentum fluid from the central region of the jet to the colder, lower momentum region of the coflow and vice versa. This increases the $z$-ward transport of $x$ momentum as well as increasing the mixing of hot and cool fluid. The results also show that the initial configuration of "necklace" vortices on both the upper and lower edges of the jet, is generally unstable. The inner most vortices interact strongly with each other, tend to be displaced, and are dissipated, starting with the next to the outermost and proceeding inward. The outer most vortices tend to become stronger and move toward the top and bottom of the channel. In contrast, if the initial configuration is a set of "trailing" vortices on both the upper and lower edges of the jet, it is stable.

Finally, it was shown that the results of the simulations were in very good qualitative agreement with visualizations of the flow induced by tabs on the edges of jets. This was true for both "necklace" and "trailing" type vortices generated by different orientations of the tabs in the experiments.

Additional simulations of the interactions of streamwise vortices with jets of circular cross section and jets exiting from lobed nozzles are in progress. The results of these calculations will be reported later. 


\section{References}

[1] J.M. Seiner and E.A. Krejsa, "Supersonic jet noise and the High Speed Civil Transport," AIAA Paper 89-2358, 1989.

[2] W.M. Presz, Jr, "Mixer/Ejector Noise Suppressors," AIA A Paper 91-2243, 1991.

[3] T.G. Tillman, W.P. Patrick and R.W. Peterson, "Enhanced mixing of supersonic jets," J. Propulsion, 7, 1006 (1991).

[4] K.K. Ahuja and W.H. Brown, "Shear flow control by mechanical tabs," AIAA Paper 89-0994, 1989.

[5] M. Samimy, K.B.M.Q. Zaman, and M.F. Reeder, "Effect of tabs on the flow and noise field of an axisymmetric jet," AIAA J., 31, 609 (1993).

[6] K.B.M.Q. Zaman, "Streamwise vorticity generation and mixing enhancement in free jets by delta-tabs," AIAA Paper 93-3253, 1993.

[7] K.K. Ahuja, "Mixing enhancement and jet noise reduction through tabs plus ejectors," AIAA Paper 93-434\%, 1993.

[8] K.B.M.Q. Zaman, M.F. Reeder and M. Samimy, "Control of an axisymmetric jet using vortex generators," Phys. Fluids, 6, 778 (1994).

[9] L.J.S. Bradbury and A.H. Khadem, "The distortion of a jet by tabs," J. Fluid Mechanics, $70,801(1975)$.

[10] F.F. Grinstein, E.J. Gutmark, T.P. Parr, D.M. Hanson-Parr and U. Obeysekare, "Streamwise and spanwise vortex interaction in an axisymmetric jet. A computational and experimental study," Physics of Fluids, 8, 1515 (1996).

[11] R. Peyret and T.D. Taylor, Computational Methods for Fluid Flow, Springer-Verlag, New York Heidelberg Berlin, 1983.

[12] C.E. Grosch and T.L. Jackson, "Ignition and structure of a laminar diffusion flame in a compressible mixing layer," Physics of Fluids, A 3, 3087 (1991).

[13] F. Kozusko, D.G. Lasseigne, C.E. Grosch and T.L. Jackson, "The stability of compressible mixing layers in binary gases," Physics of Fluids, 8, 1954 (1996).

[14] D. Papamoschou and A. Roshko, "The compressible turbulent shear layer: An experimental study," J. Fluid Mechanics, 197, 453 (1989). 


\section{Table 1: Inflow Conditions for Simulation Runs}

\begin{tabular}{|c|c|c|c|c|c|c|c|c|c|c|c|}
\hline \multirow[b]{2}{*}{ Case } & \multirow[b]{2}{*}{ Tabs } & \multicolumn{5}{|c|}{ Jet } & \multicolumn{4}{|c|}{ Coflow } & \multirow[b]{2}{*}{$M_{c}$} \\
\hline & & $\begin{array}{r}U \\
(\mathrm{~m} / \mathrm{s})\end{array}$ & $\begin{array}{r}T \\
\left(K^{\circ}\right)\end{array}$ & $\begin{array}{r}P \\
\text { (bar) }\end{array}$ & $M$ & $\operatorname{Re} \times 10^{-5}$ & $\begin{array}{r}U \\
(\mathrm{~m} / \mathrm{s})\end{array}$ & $\begin{array}{r}T \\
\left(K^{\circ}\right)\end{array}$ & $\begin{array}{r}P \\
\text { (bar) }\end{array}$ & $M$ & \\
\hline 1 & No & 695.0 & 490.0 & 1.0133 & 1.566 & 1.305 & 220.0 & 300.0 & 1.0133 & 0.634 & 0.601 \\
\hline 2 & No & 695.0 & 490.0 & 0.9322 & 1.566 & 1.201 & 220.0 & 300.0 & 1.0133 & 0.634 & 0.601 \\
\hline 3 & 6 & 695.0 & 490.0 & 0.9322 & 1.566 & 1.201 & 220.0 & 300.0 & 1.0133 & 0.634 & 0.601 \\
\hline 4 & No & 950.0 & 490.0 & 1.0133 & 2.141 & 2.473 & 50.0 & 250.0 & 1.0133 & 0.158 & 1.183 \\
\hline 5 & No & 695.0 & 490.0 & 1.0133 & 1.566 & 1.635 & 100.0 & 250.0 & 1.0133 & 0.316 & 0.782 \\
\hline 6 & 4 & 695.0 & 490.0 & 1.0133 & 1.566 & 1.305 & 220.0 & 300.0 & 1.0133 & 0.634 & 0.601 \\
\hline 7 & 2 & 695.0 & 490.0 & 1.0133 & 1.566 & 1.305 & 220.0 & 300.0 & 1.0133 & 0.634 & 0.601 \\
\hline $8^{*}$ & 6 & 695.0 & 490.0 & 1.0133 & 1.566 & 1.305 & 220.0 & 300.0 & 1.0133 & 0.634 & 0.601 \\
\hline 9 & 8 & 695.0 & 490.0 & 1.0133 & 1.566 & 1.305 & 220.0 & 300.0 & 1.0133 & 0.634 & 0.601 \\
\hline
\end{tabular}

Note that ${ }^{*}$ denotes the case with periodic boundary conditions in $y$. 


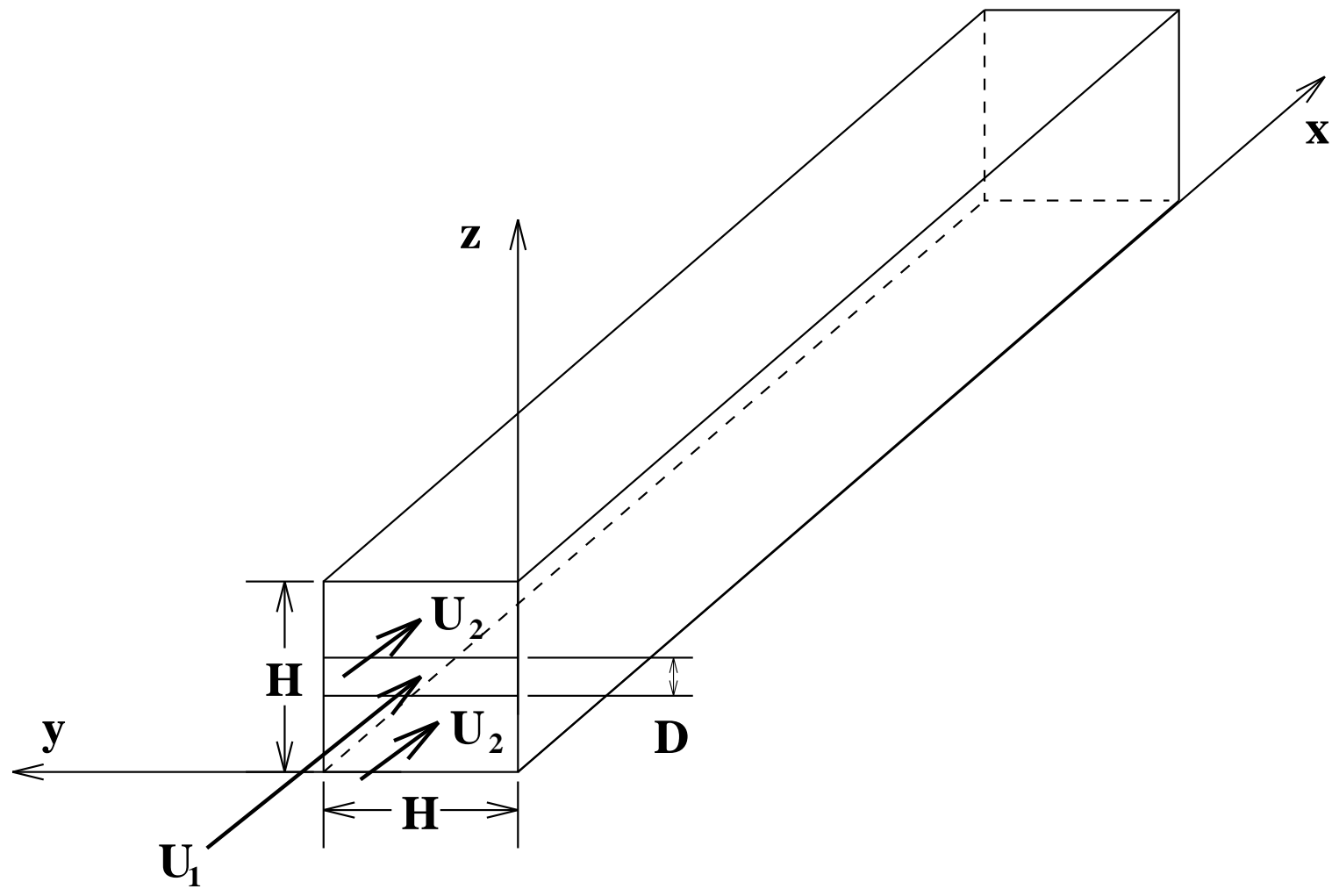

Figure 1: Schematic of the channel and coordinate system. 


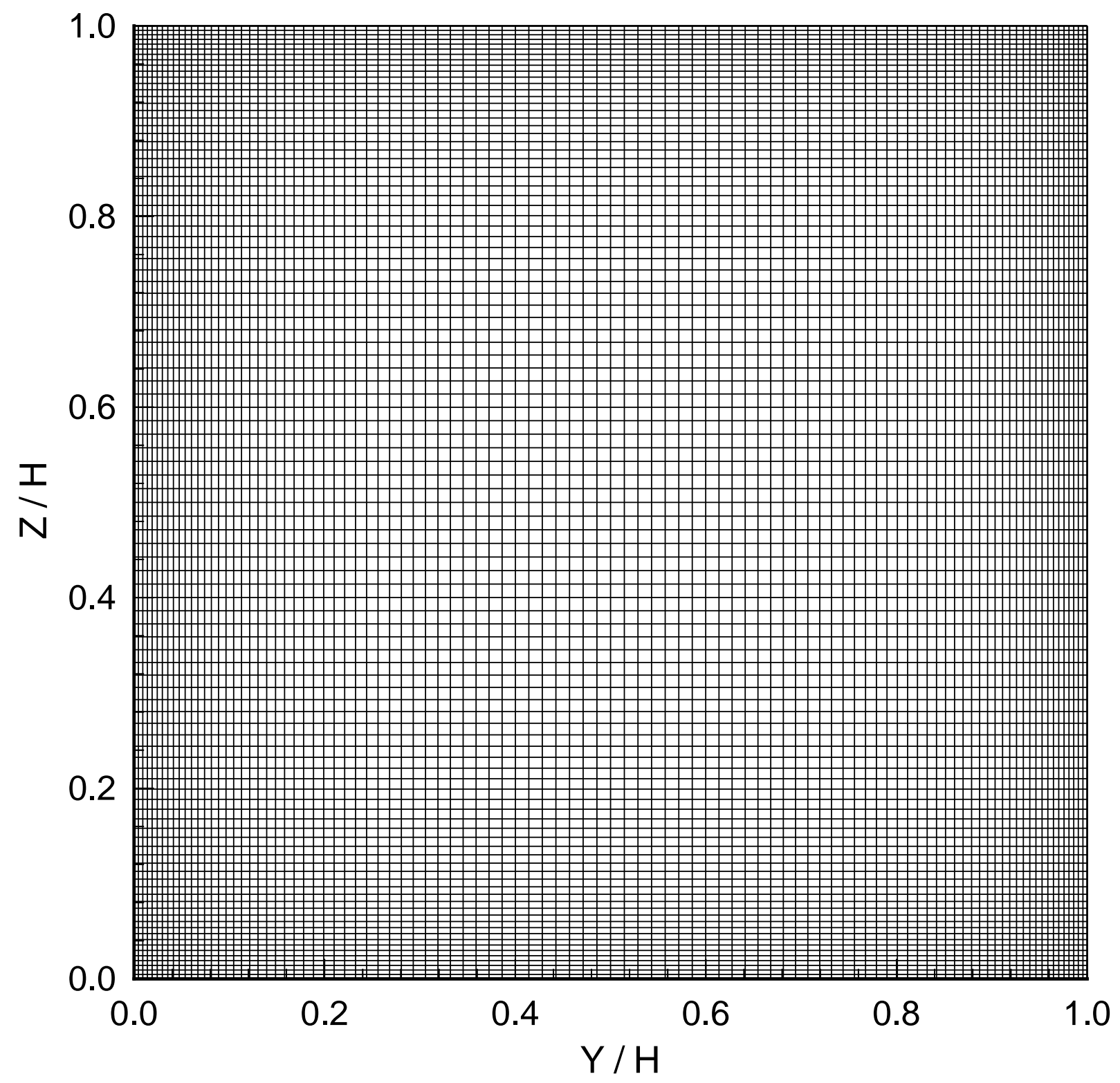

Figure 2: Grid on the $Y-Z$ plane with $N_{y}=101$ and $N_{z}=101$. 

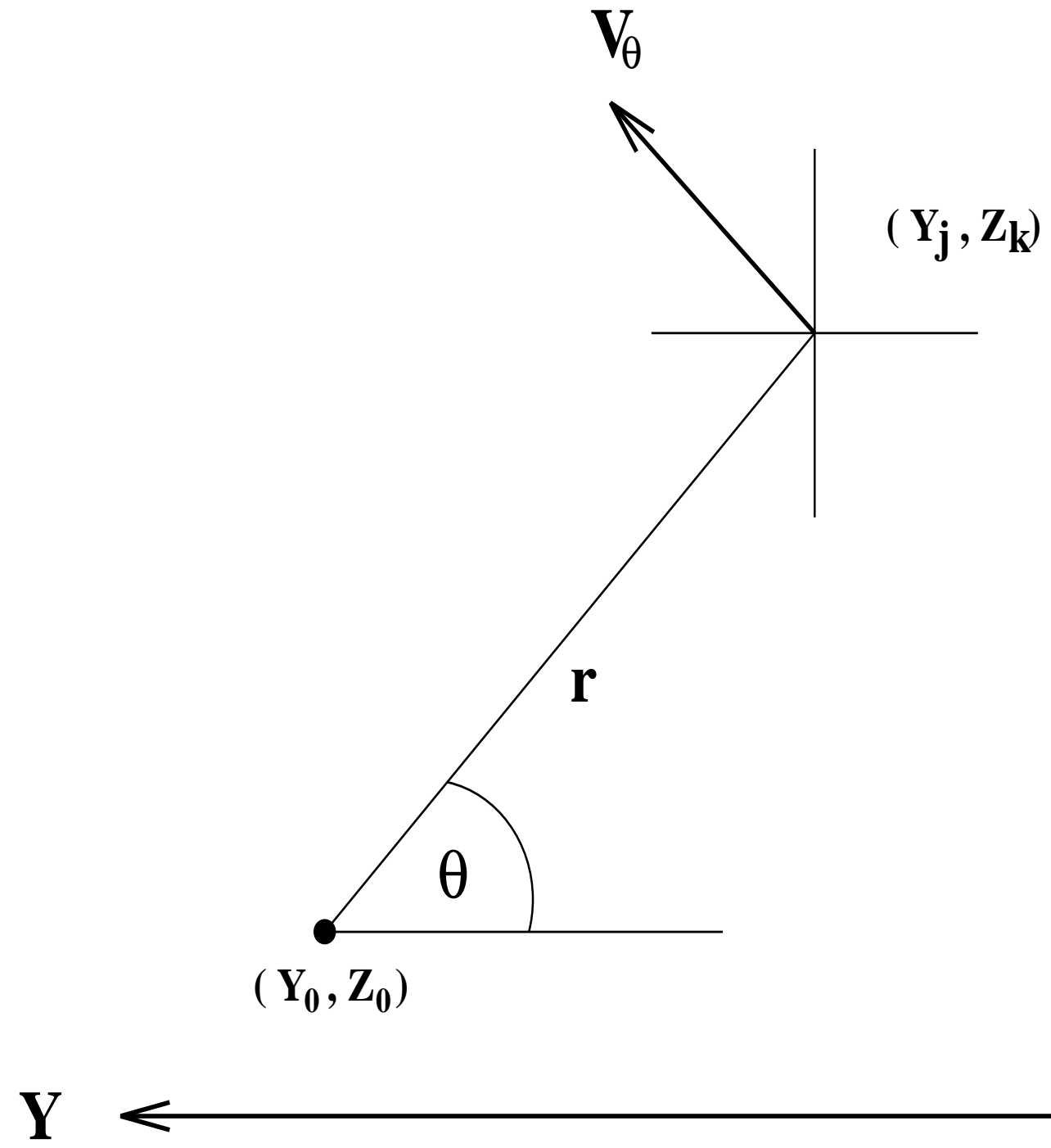

Figure 3: Sketch illustrating the basic geometry of the model vortices. The point $\left(Y_{o}, Z_{o}\right)$ is the center of one of the vortices, the point $\left(Y_{j}, Z_{k}\right)$ is any grid point on the inflow boundary, and the radial distance between these points is $r$. 

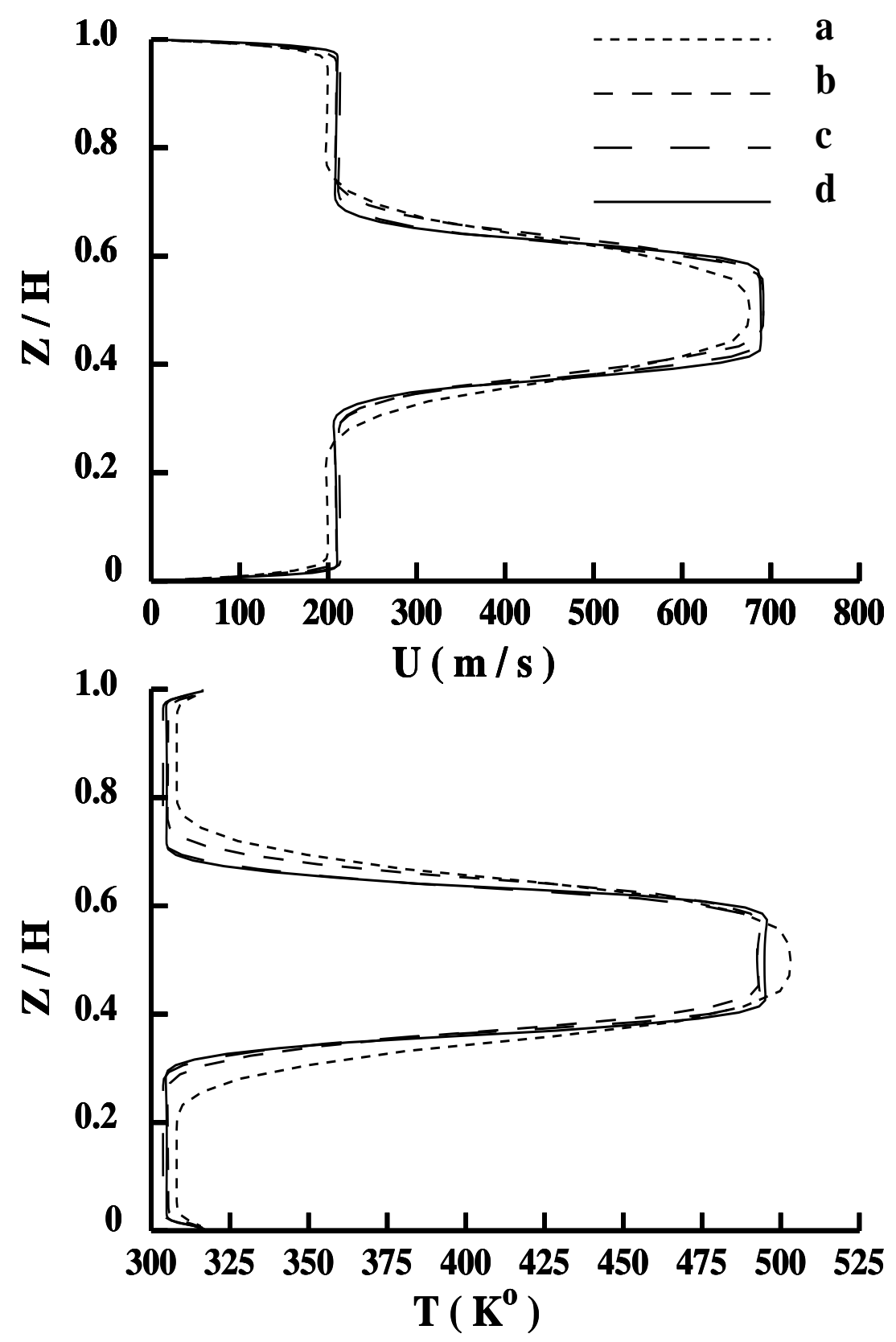

Figure 4: Profiles of the streamwise velocity component, $U$, and temperature, $T$, as a function of the dimensionless height, $Z / H$, at $Y / H=0.5$ and $X / D=6.0$ as computed on different grids. The grids in the $(Y, Z)$ plane are: $[\mathrm{a}](51 \times 51)$; [b] $(76 \times 76) ;[\mathrm{c}](101 \times 101)$; [d] $(126 \times 126)$. 


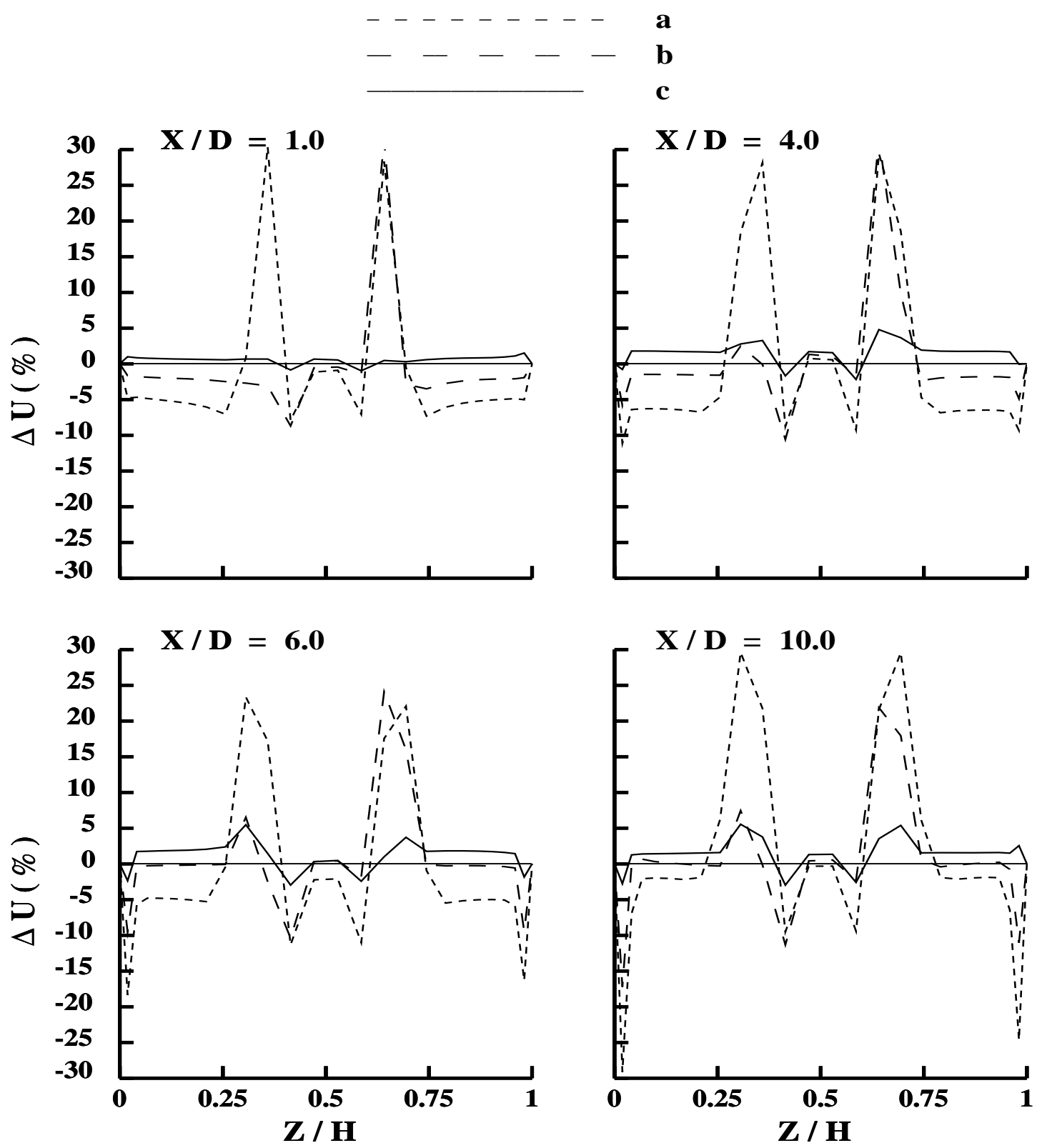

Figure 5: Profiles of the differences, $\Delta U$, in the streamwise velocity component as a function of the dimensionless height, $Z / H$, at $Y / H=0.5$ and $X / D=6.0 . \Delta U$ is defined as the velocity on a particular coarse grid minus the velocity on the finest, $(126 \times 126)$ grid. The notation is: $\left(N_{y} \times N_{z}\right)=[\mathrm{a}](51 \times 51) ;[\mathrm{b}](76 \times 76) ;[\mathrm{c}](101 \times 101)$. In all cases $N_{x}=145$. 

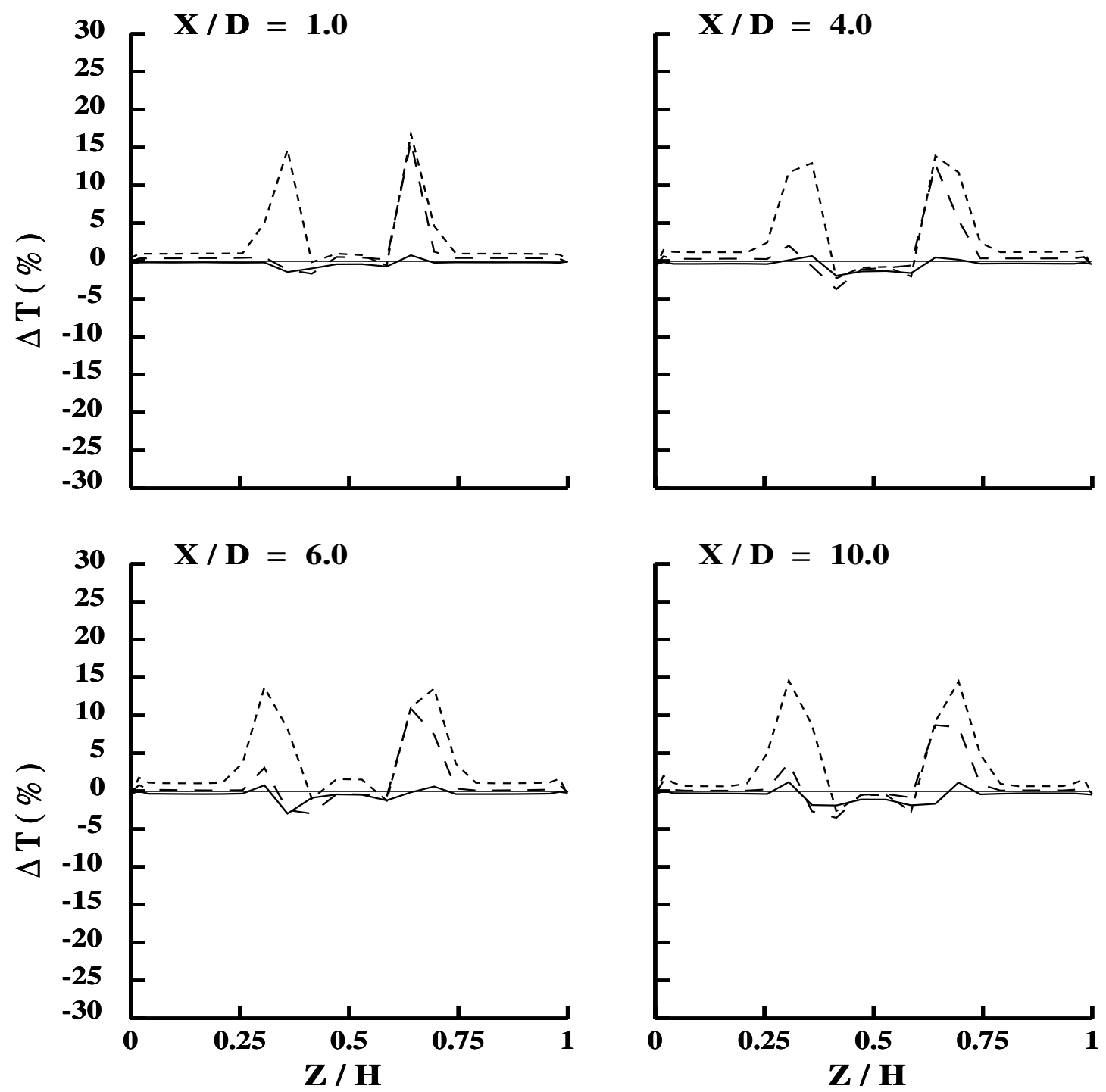

Figure 6: Profiles of the differences, $\Delta T$, in the temperature field as a function of the dimensionless height, $Z / H$, at $Y / H=0.5$ and $X / D=6.0 . \Delta T$ is defined as the temperature on a particular coarse grid minus the temperature on the finest, $(126 \times 126)$ grid. The notation is the same as in Figure 5. 


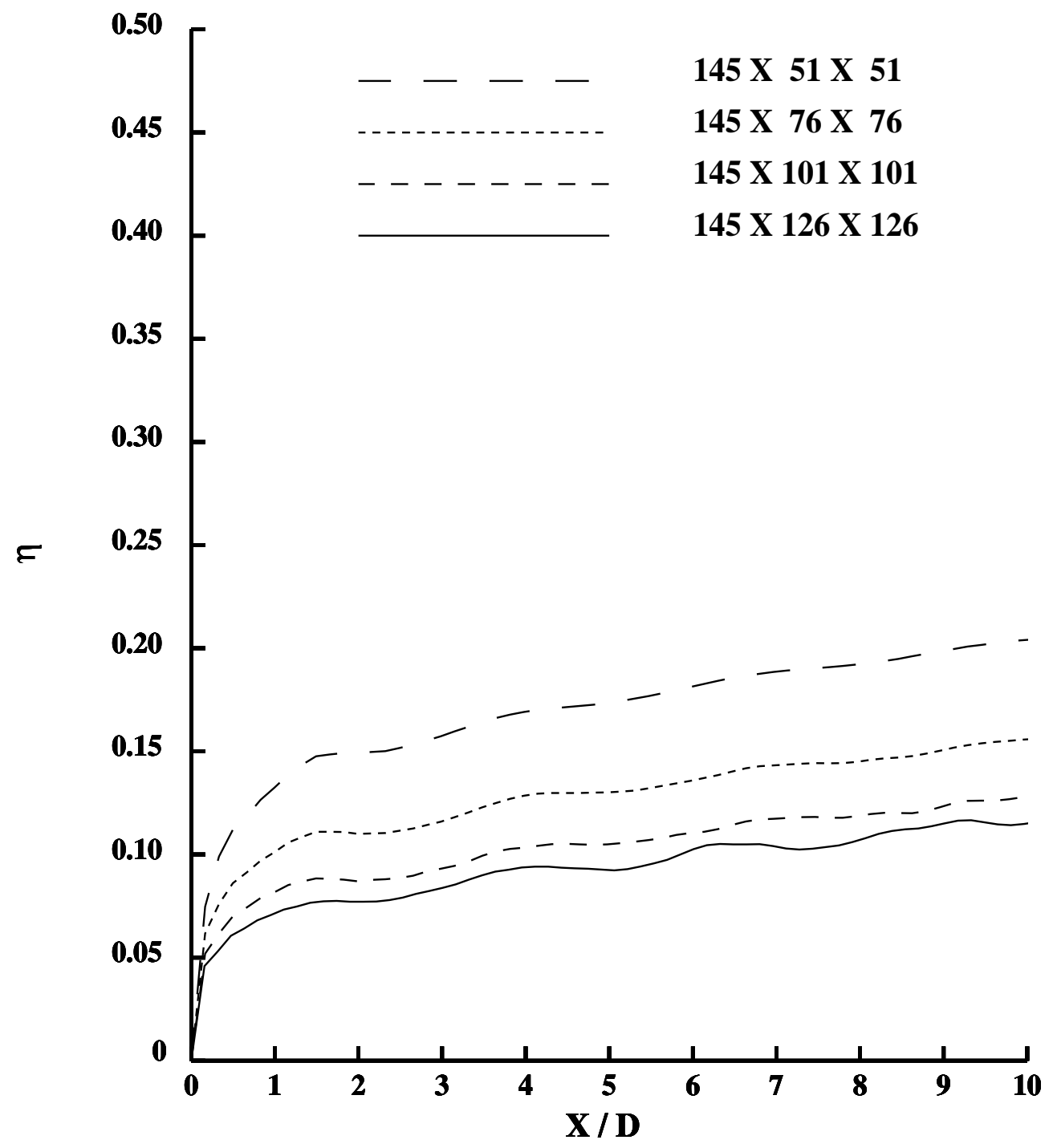

Figure 7: Variation of the mixing parameter, $\eta$, as a function of $X / D$ on different $\left(N_{y} \times N_{z}\right)$ grids. In all cases $N_{x}=145$. 

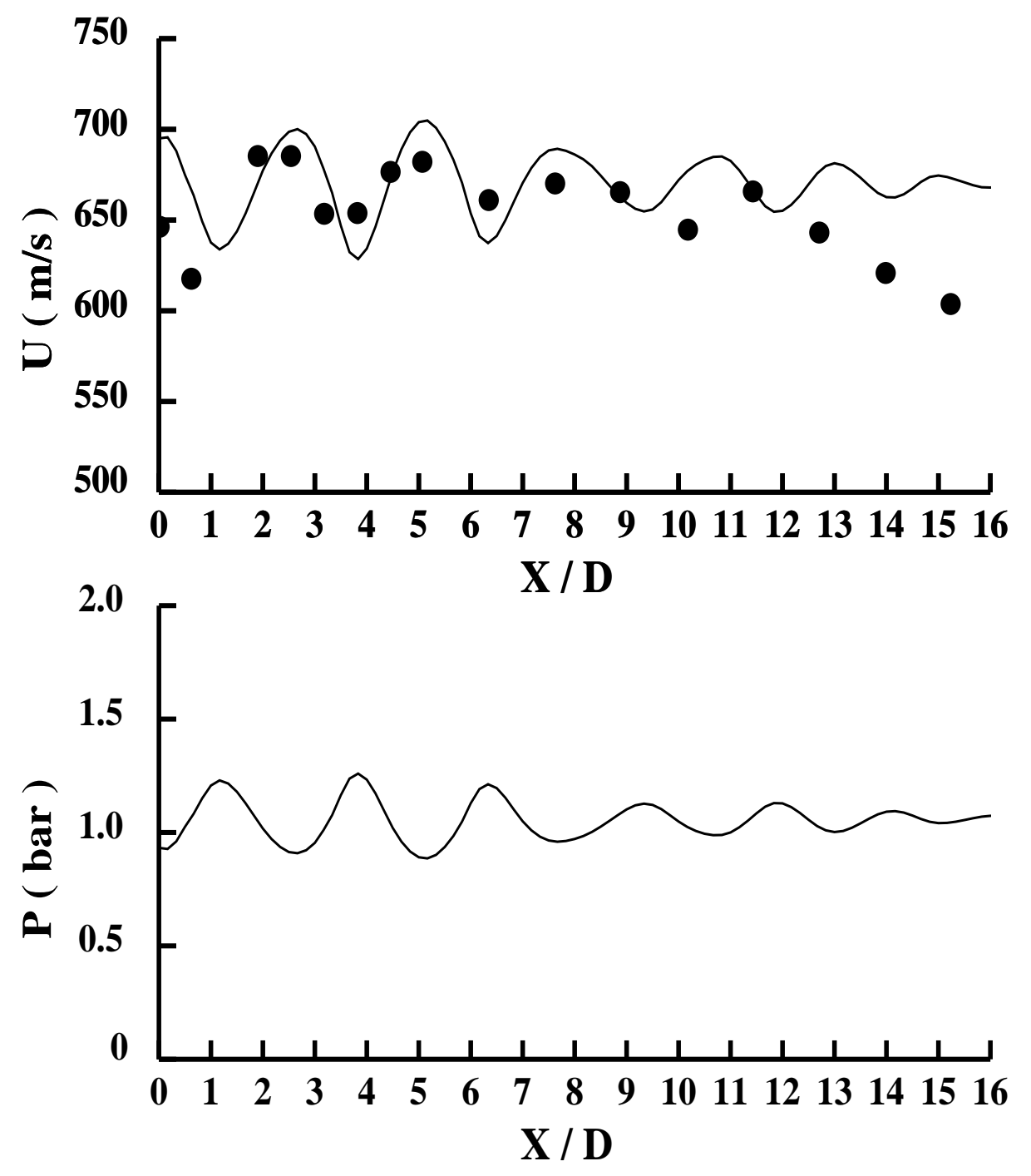

Figure 8: Plot of the computed jet centerline velocity and pressure for the inflow conditions of Case 2 ( Table 1 ) with no tabs. The measured values of the velocity on the jet centerline are denoted by $\bullet$. 

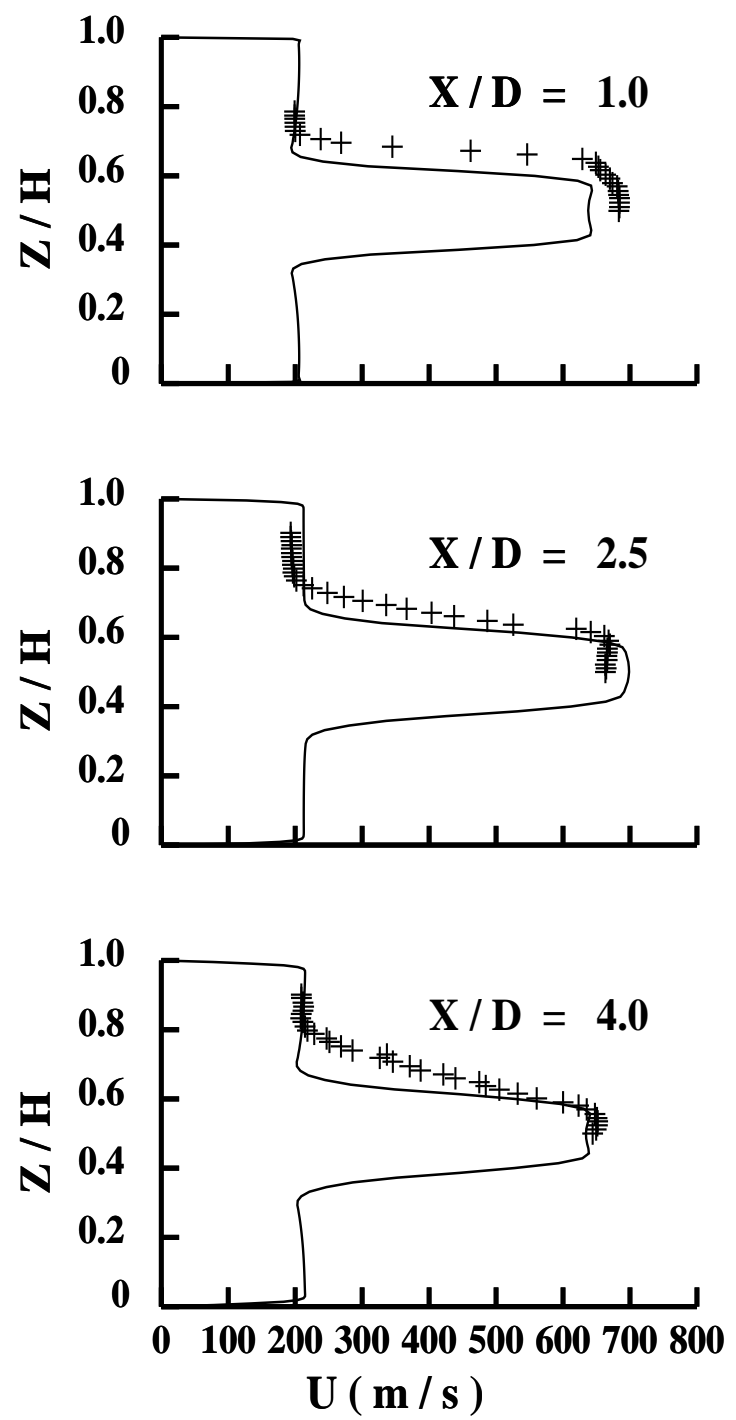

Figure 9: The calculated profiles of $U$ for the inflow conditions of Case 2 ( Table 1) with no tabs. The + denote the measured values. 


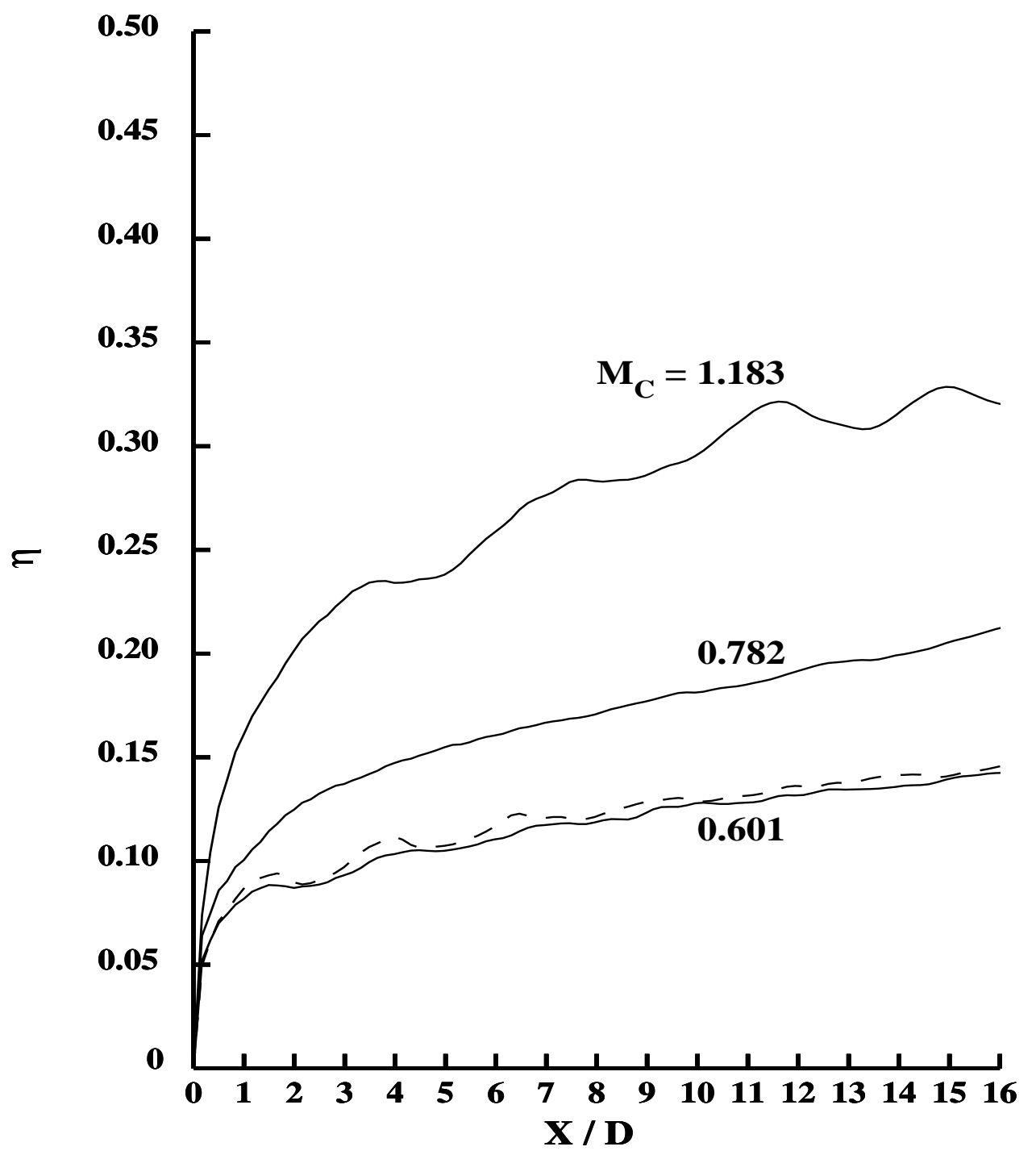

Figure 10: Variation of the mixing parameter $\eta$ with downstream distance for various values of the convective Mach number, $M_{C}$. These calculations were run with no tabs on the edge of the jet. Values of the flow variables at input are given in Table 1. The dashed curve shows results for Case 2; at $M_{C}=0.601$ but with a slightly reduced pressure in the jet at inflow. 


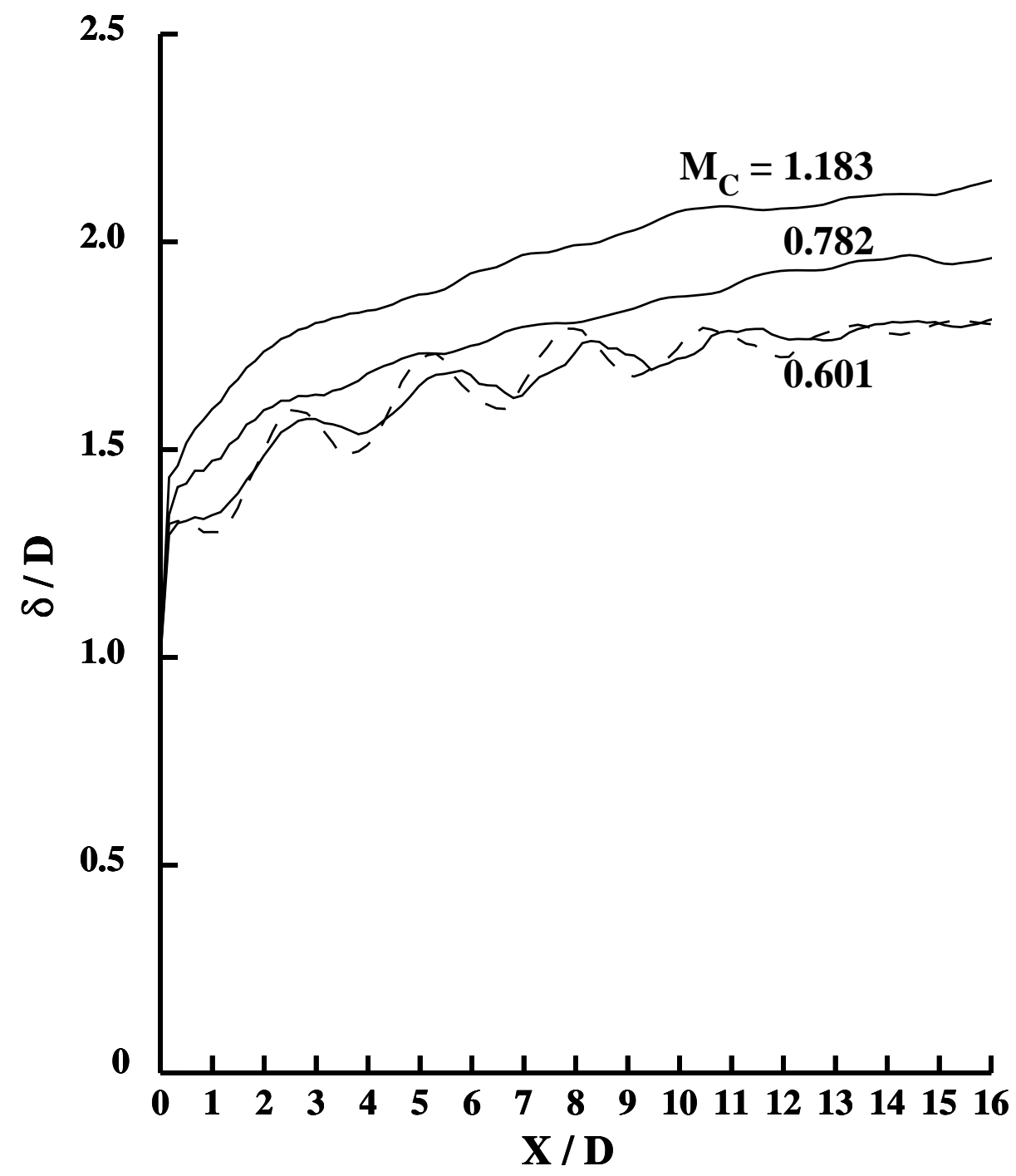

Figure 11: Variation of the jet thickness, $\delta / D$, with downstream distance for various values of the convective Mach number, $M_{C}$. These calculations were run with no tabs on the edge of the jet. Values of the flow variables at input are given in Table 1 . The dashed curve shows results for Case 2; at $M_{C}=0.601$ but with a slightly ( $8 \%$ ) reduced pressure in the jet at inflow. 

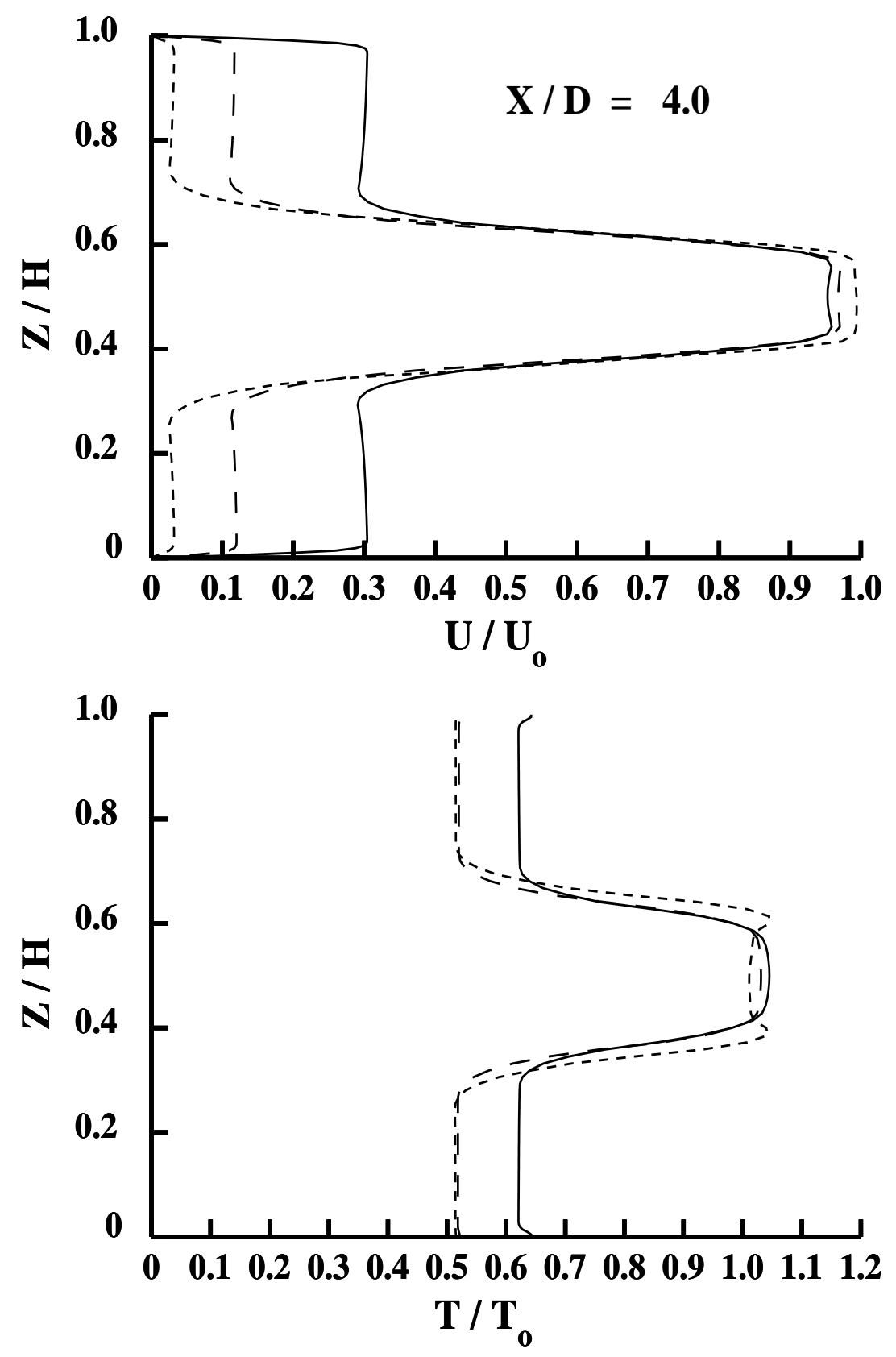

Figure 12: Profiles of the streamwise normalized velocity component, $U / U_{o}$, and temperature, $T / T_{o}$, as a function of the dimensionless height, $Z / H$, at $Y / H=0.5$ and $X / D=4.0$ at different values of $M_{C}$. In each case the velocity and temperature were scaled by the values of the velocity $\left(U_{o}\right)$ and temperature $\left(T_{o}\right)$ of the jet at the inflow boundary. The solid line denotes results for $M_{C}=0.601$; the long dash line for $M_{C}=0.782$; and the short dash line for $M_{C}=1.183$. 


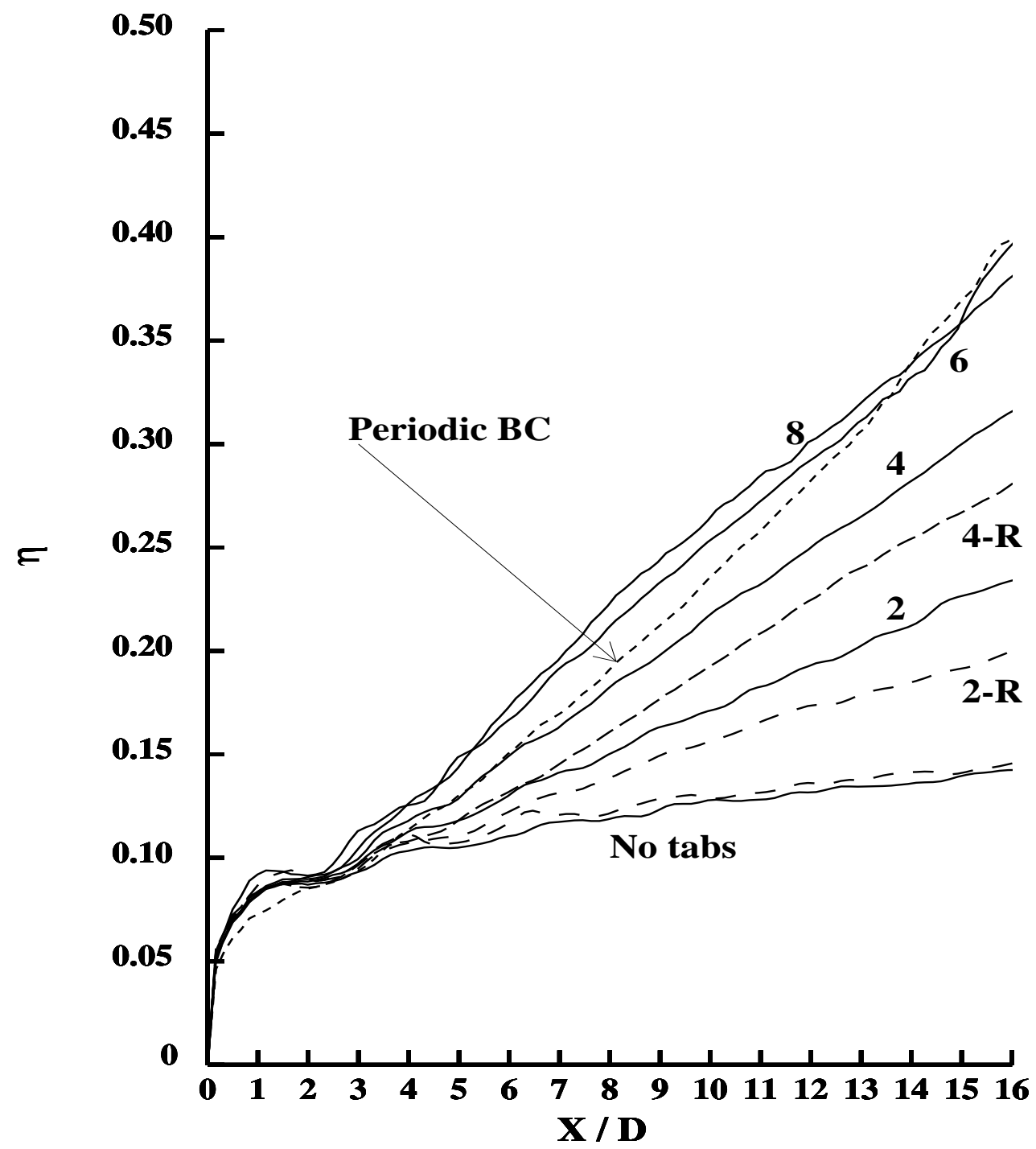

Figure 13: The solid curves are the variation of the mixing parameter $\eta$ with downstream distance for zero (Case 1), two (Case 7), four (Case 6), six (Case 3) and eight tabs (Case 9) all at $M_{C}=0.601$. Also, results for the case of six tabs and periodic side wall boundary conditions are shown, again at $M_{C}=0.601$. All of these results were obtained using pairs of "necklace" vortices to simulate the tabs. The dashed curve labeled $4-T$ is the result with four tabs (Case 6) but with pairs of "trailing" vortices used to simulate the tabs. The dashed curve labeled $2-T$ is the result with two tabs (Case 7 ) but with pairs of "trailing" vortices used to simulate the tabs. The dashed curve labeled No tabs was obtained using the inflow data of Case 2, i.e. with a slightly reduced pressure. 


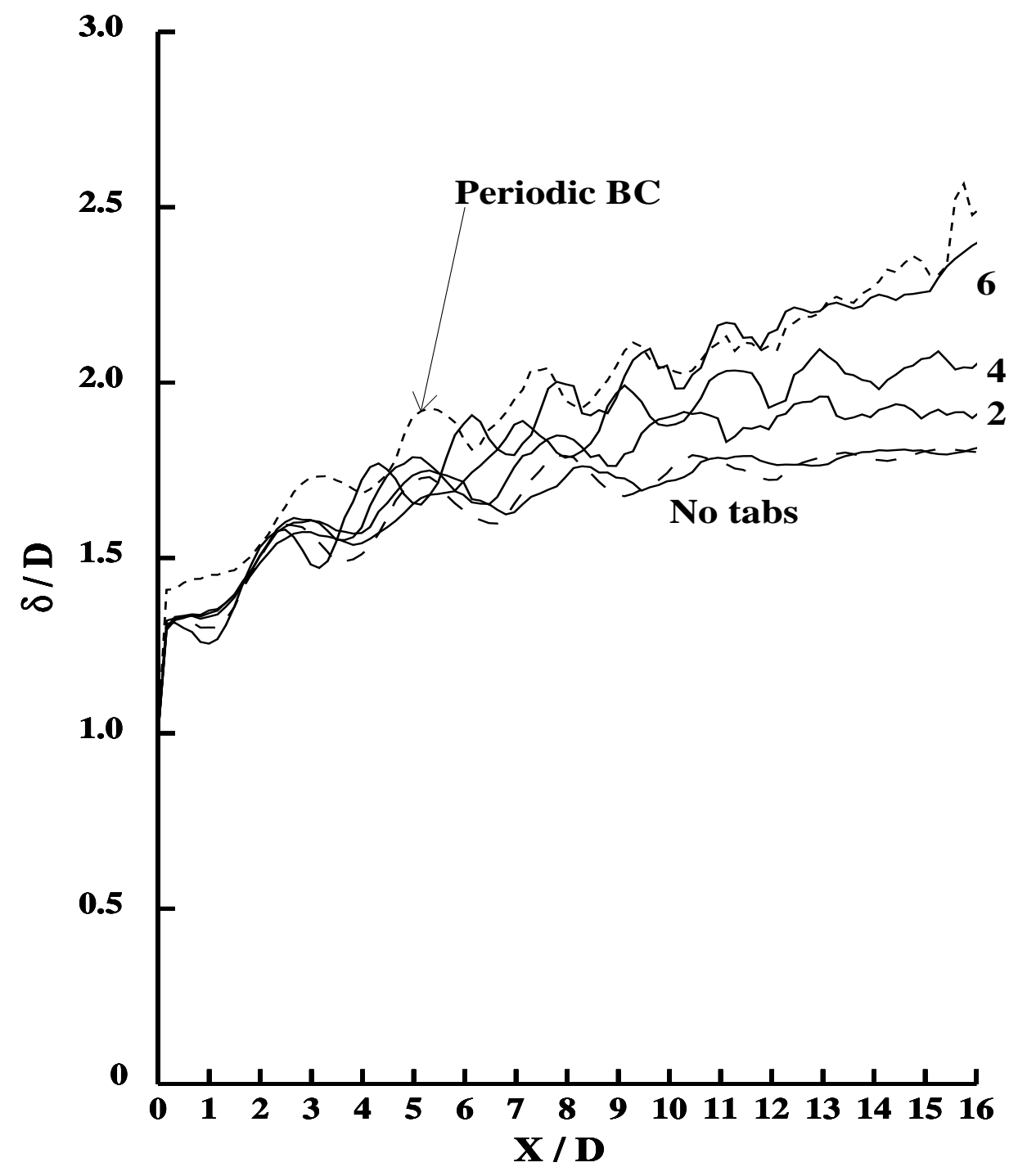

Figure 14: Variation of the jet thickness $\delta / D$ with downstream distance with zero (Case 1), two (Case 7), four (Case 6), six tabs (Case 3) and eight tabs (Case 9) all at $M_{C}=0.601$. Also, results for the case of six tabs and periodic side wall boundary conditions are shown, again at $M_{C}=0.601$. The dashed curve labeled No tabs was obtained using the inflow data of Case 2 . 


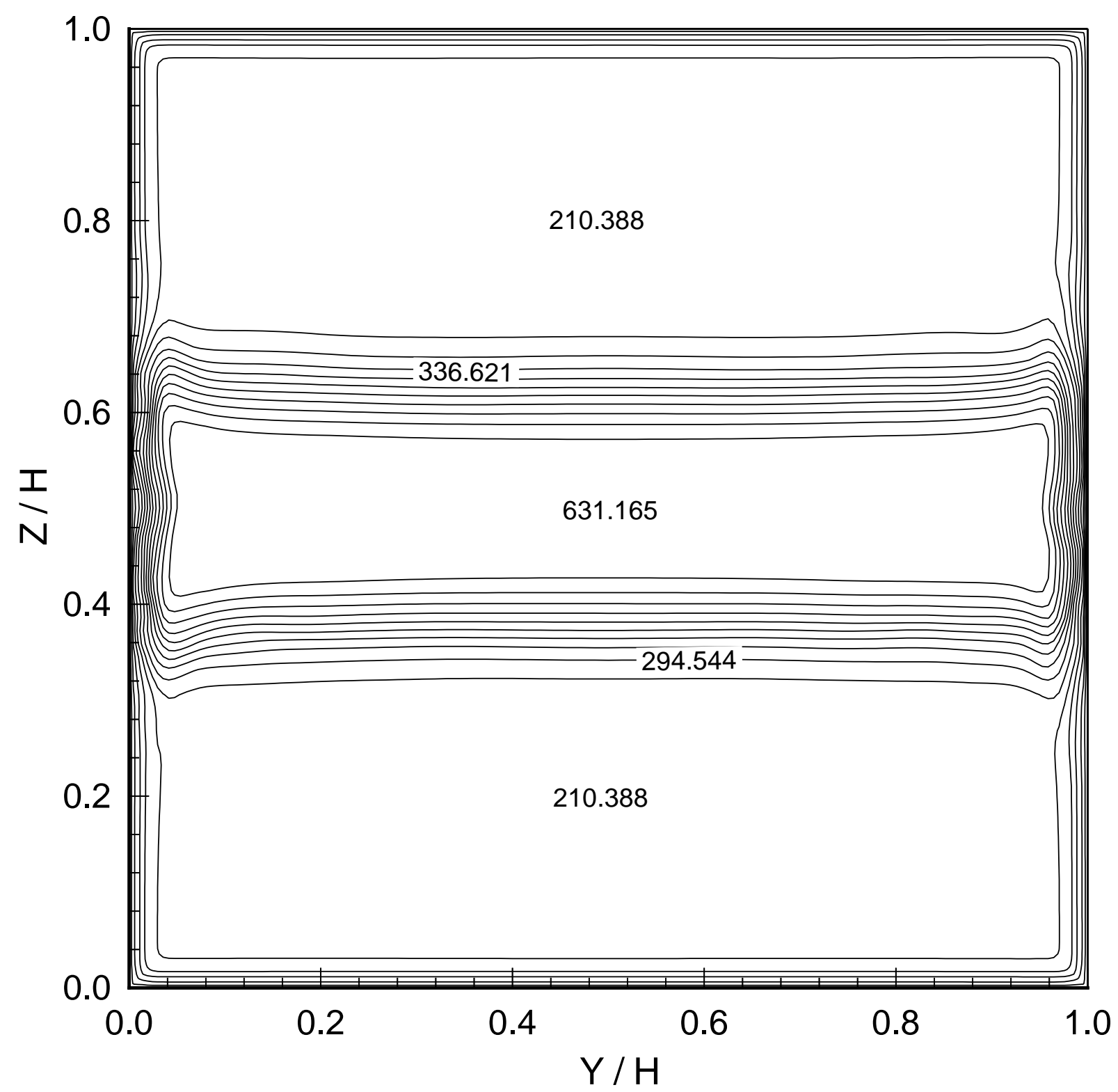

Figure 15: Contours of $U$ on the $(y, z)$ plane at $x / D=14.0$. The inflow conditions are those of Case 2 ( Table 1 ) and there are no tabs. Contour values are in $\mathrm{m} / \mathrm{s}$. 


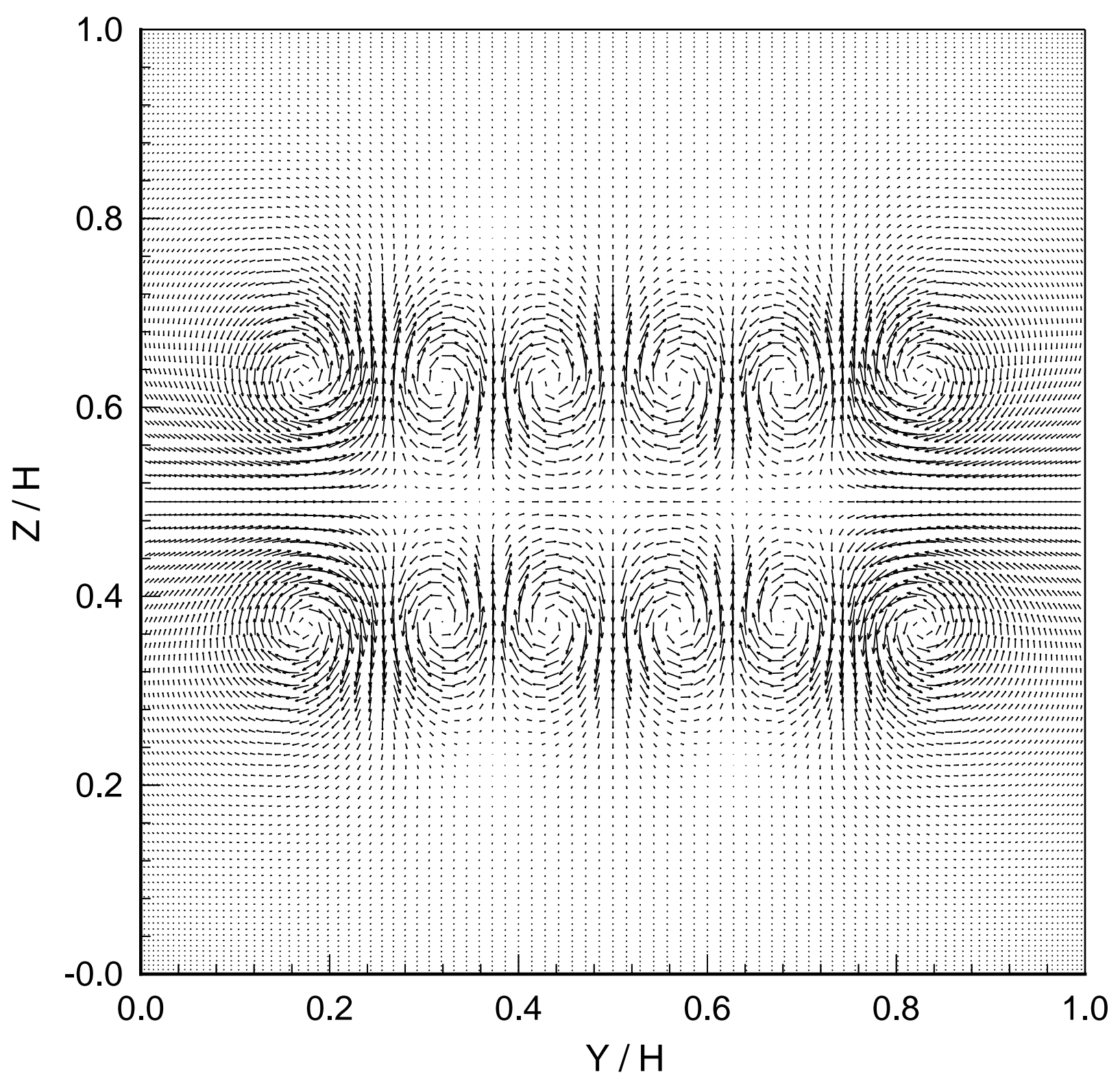

Figure 16: Vectors of $(V, W)$ on the inflow boundary, $x / D=0.0$. The maximum value of the velocity on this plane is $79.0 \mathrm{~m} / \mathrm{s}$. The inflow conditions are those of Case 2 ( Table 1 ) and there are three tabs on both the upper and lower edges of the jet at $y / H=1 / 4,1 / 2$ and $3 / 4$. The vortices are of the "necklace" type resulting from a positive $V_{0}$. 


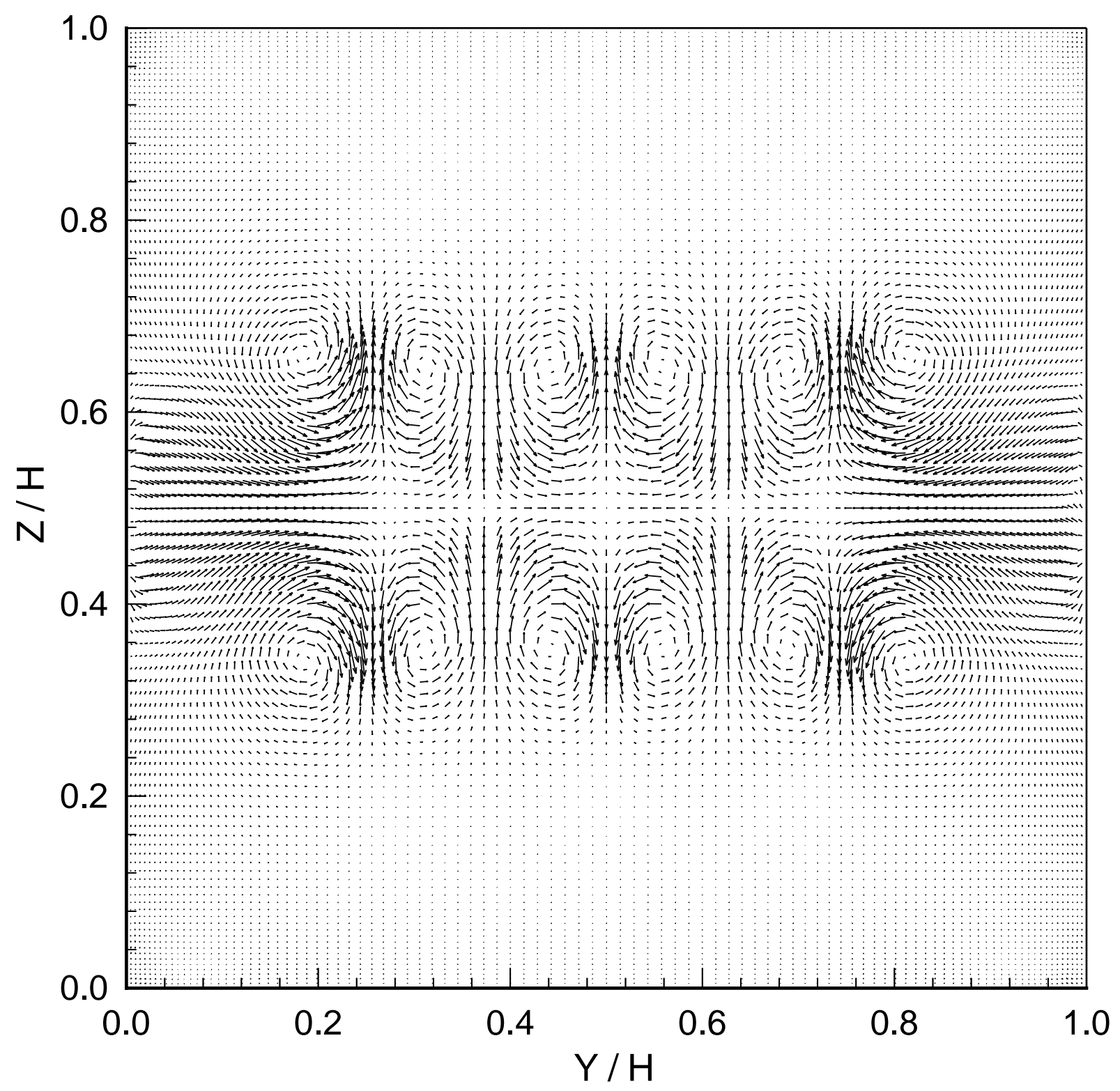

Figure 17: Vectors of $(V, W)$ on the plane at $x / D=1.0$. The maximum value of the velocity on this plane is $85.0 \mathrm{~m} / \mathrm{s}$. The inflow conditions are those of Case 2 ( Table 1 ) and there are three tabs on both the upper and lower edges of the jet at $y / H=1 / 4,1 / 2$ and $3 / 4$. The vortices are of the "necklace" type resulting from a positive $V_{0}$. 


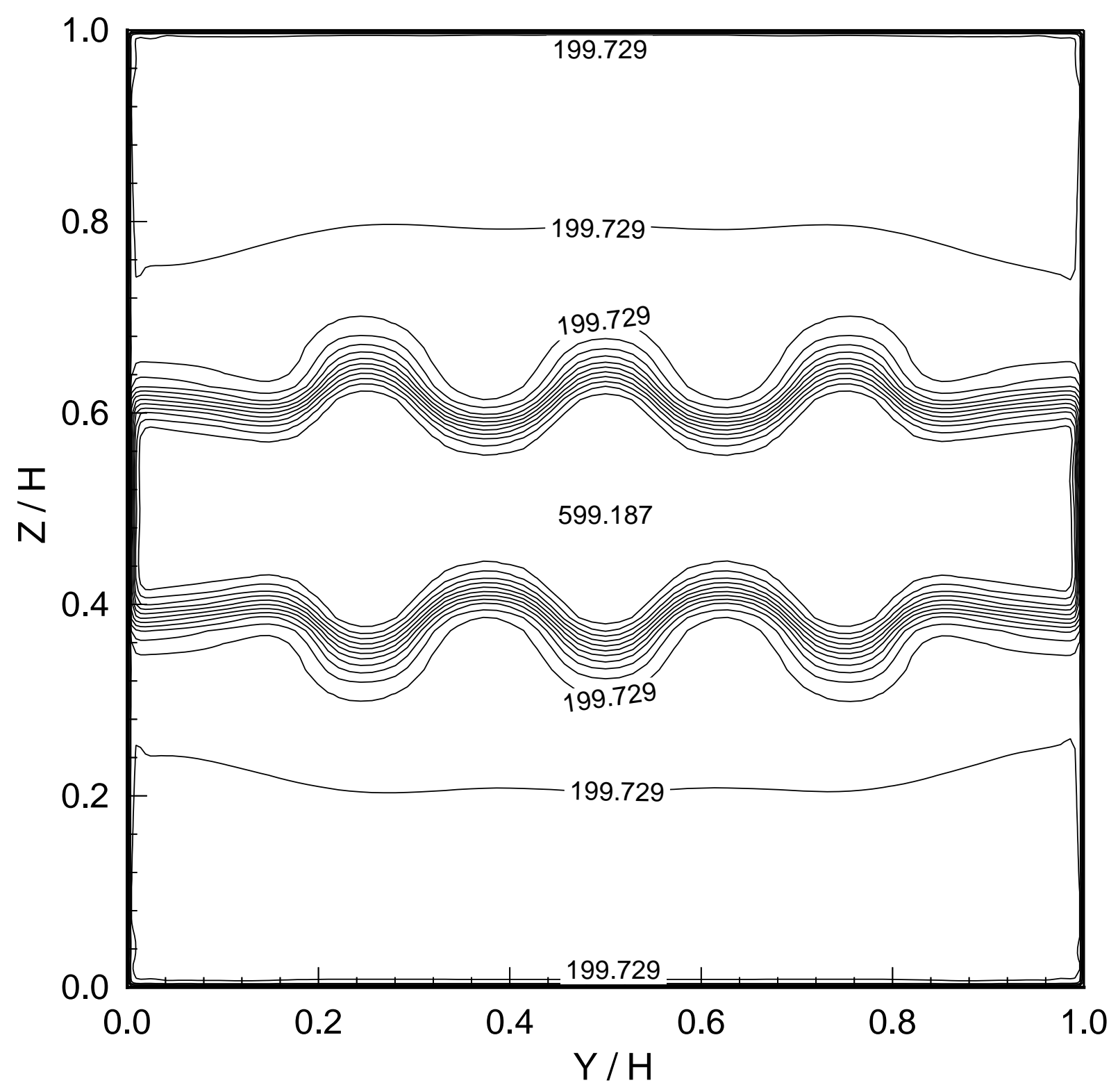

Figure 18: Contours of $U$ on the $(y, z)$ plane at $x / D=1.0$. The inflow conditions are those of Case 2 ( Table 1 ) and there are three tabs on both the upper and lower edges of the jet at $y / H=1 / 4,1 / 2$ and $3 / 4$. The vortices are of the "necklace" type resulting from a positive $V_{0}$. Contour values are in $\mathrm{m} / \mathrm{s}$. 


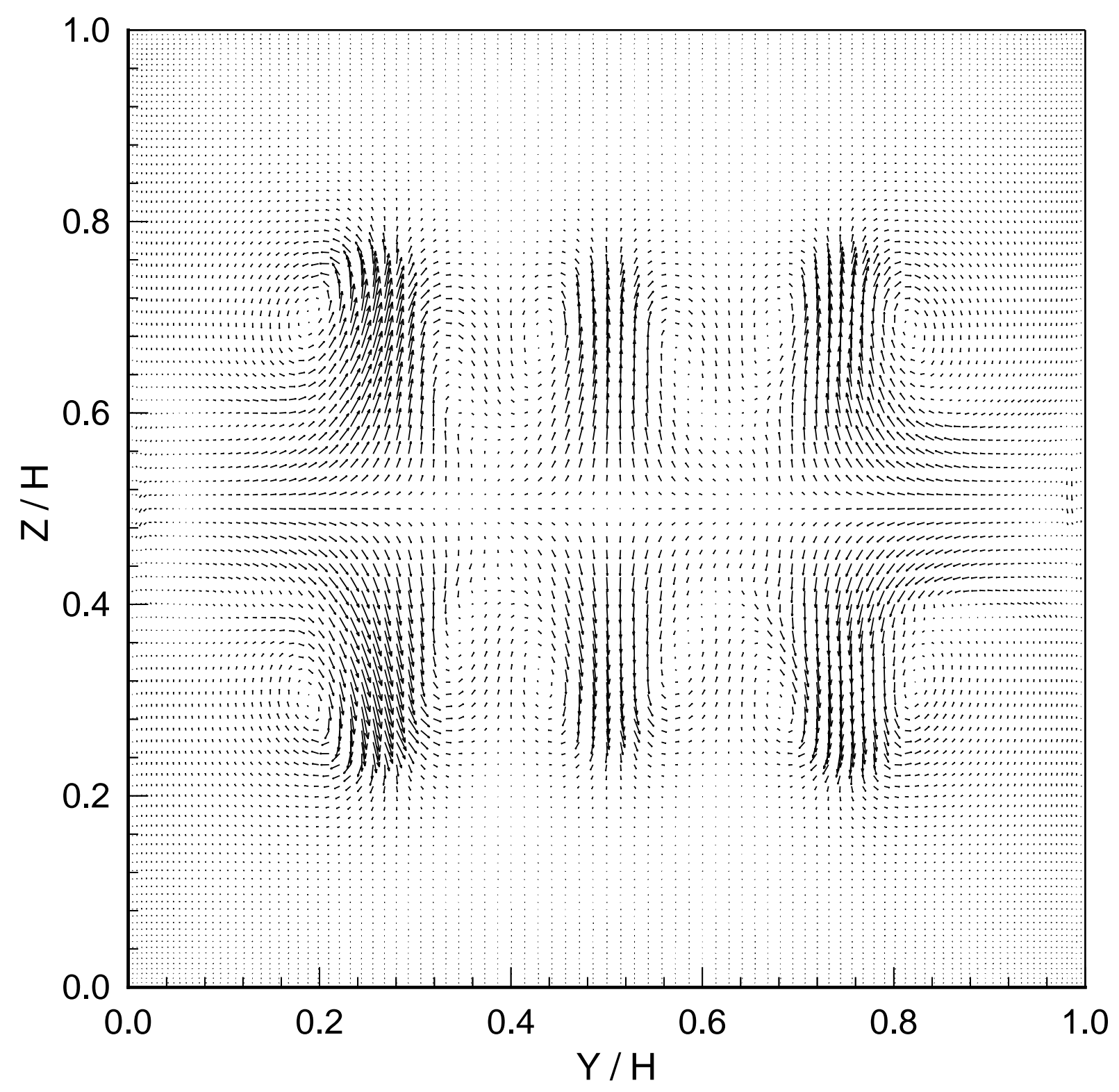

Figure 19: Vectors of $(V, W)$ on the plane at $x / D=4.0$. The maximum value of the velocity on this plane is $105.4 \mathrm{~m} / \mathrm{s}$. The inflow conditions are those of Case 2 ( Table 1 ) and there are three tabs on both the upper and lower edges of the jet at $y / H=1 / 4,1 / 2$ and $3 / 4$. The vortices are of the "necklace" type resulting from a positive $V_{0}$. 


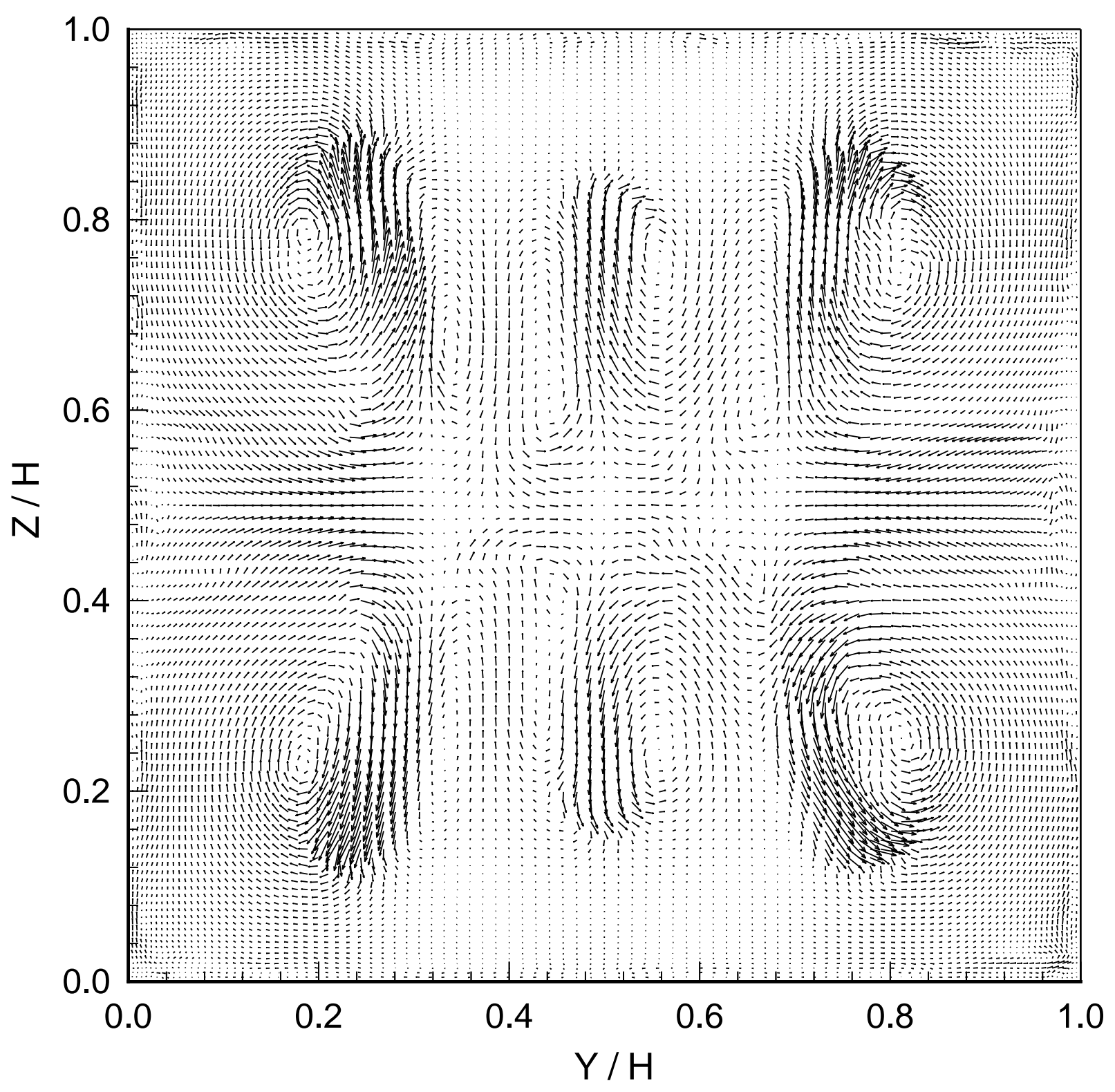

Figure 20: Vectors of $(V, W)$ on the plane at $x / D=10.0$. The maximum value of the velocity on this plane is $66.4 \mathrm{~m} / \mathrm{s}$. The inflow conditions are those of Case 2 ( Table 1 ) and there are three tabs on both the upper and lower edges of the jet at $y / H=1 / 4,1 / 2$ and $3 / 4$. The vortices are of the "necklace" type resulting from a positive $V_{0}$. 


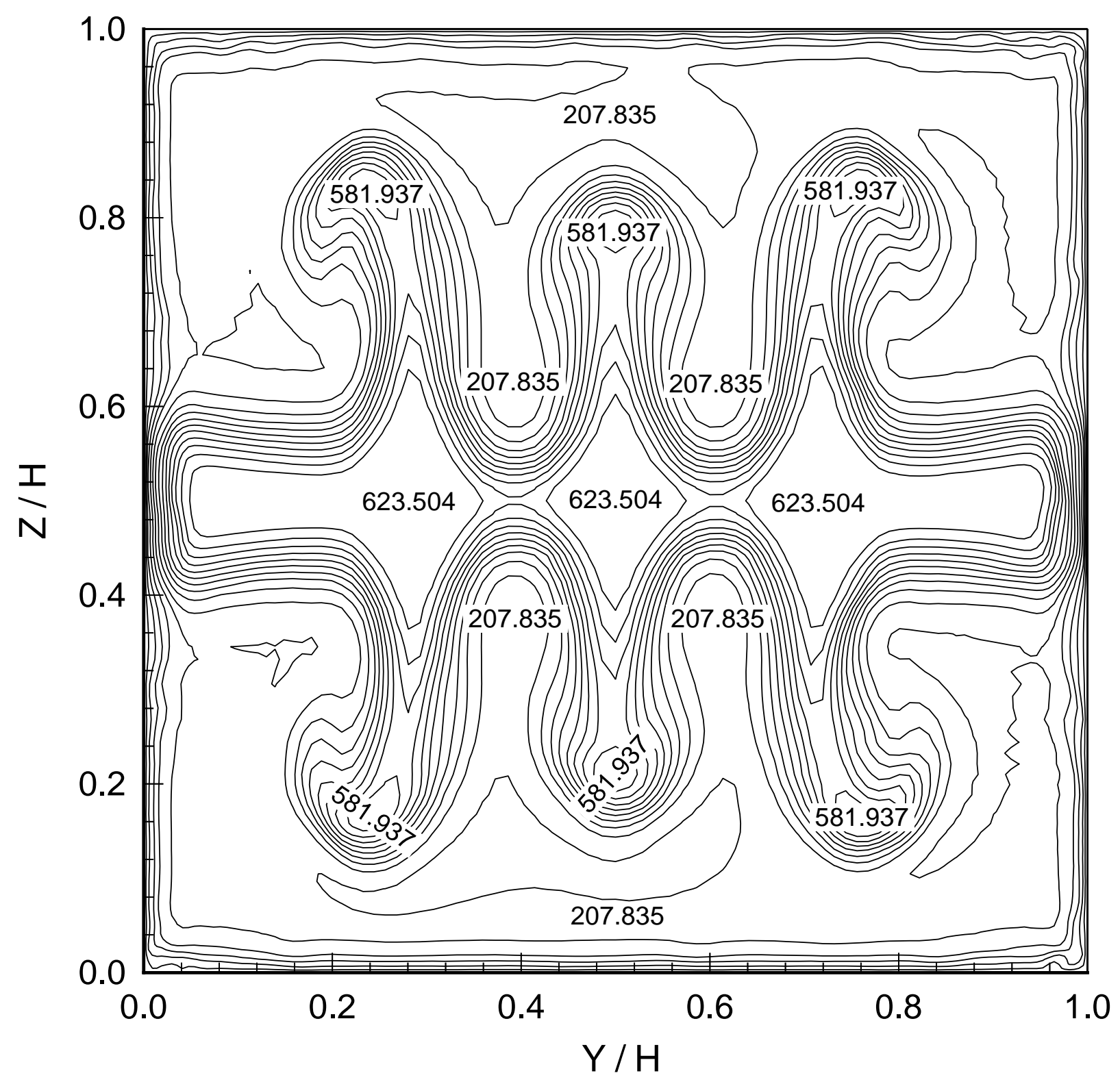

Figure 21: Contours of $U$ on the $(y, z)$ plane at $x / D=10.0$. The inflow conditions are those of Case 2 ( Table 1 ) and there are three tabs on both the upper and lower edges of the jet at $y / H=1 / 4,1 / 2$ and $3 / 4$. The vortices are of the "necklace" type resulting from a positive $V_{0}$. Contour values are in $\mathrm{m} / \mathrm{s}$. 


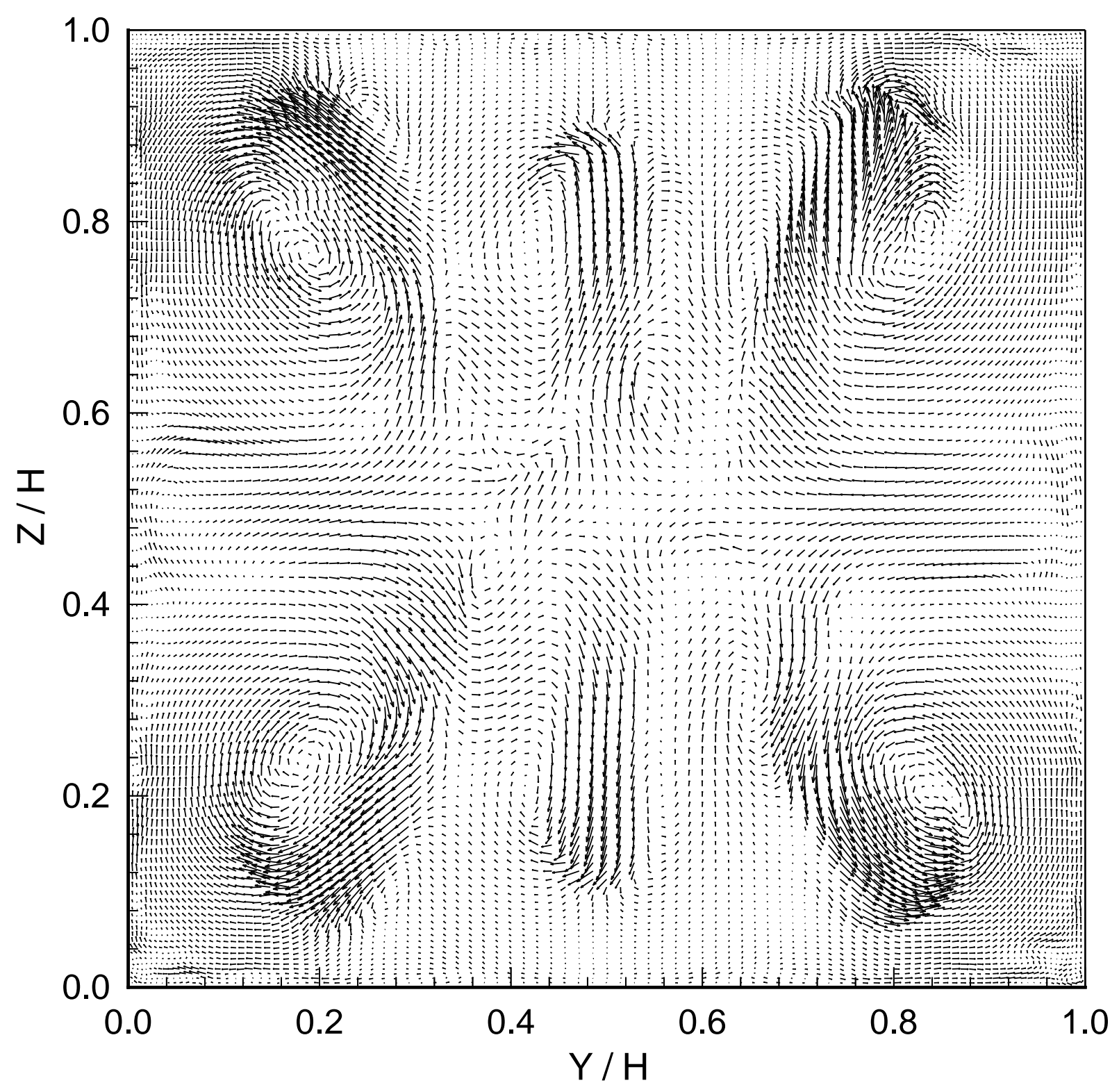

Figure 22: Vectors of $(V, W)$ on the plane at $x / D=14.0$. The maximum value of the velocity on this plane is $58.5 \mathrm{~m} / \mathrm{s}$. The inflow conditions are those of Case 2 ( Table 1 ) and there are three tabs on both the upper and lower edges of the jet at $y / H=1 / 4,1 / 2$ and $3 / 4$. The vortices are of the "necklace" type resulting from a positive $V_{0}$. 


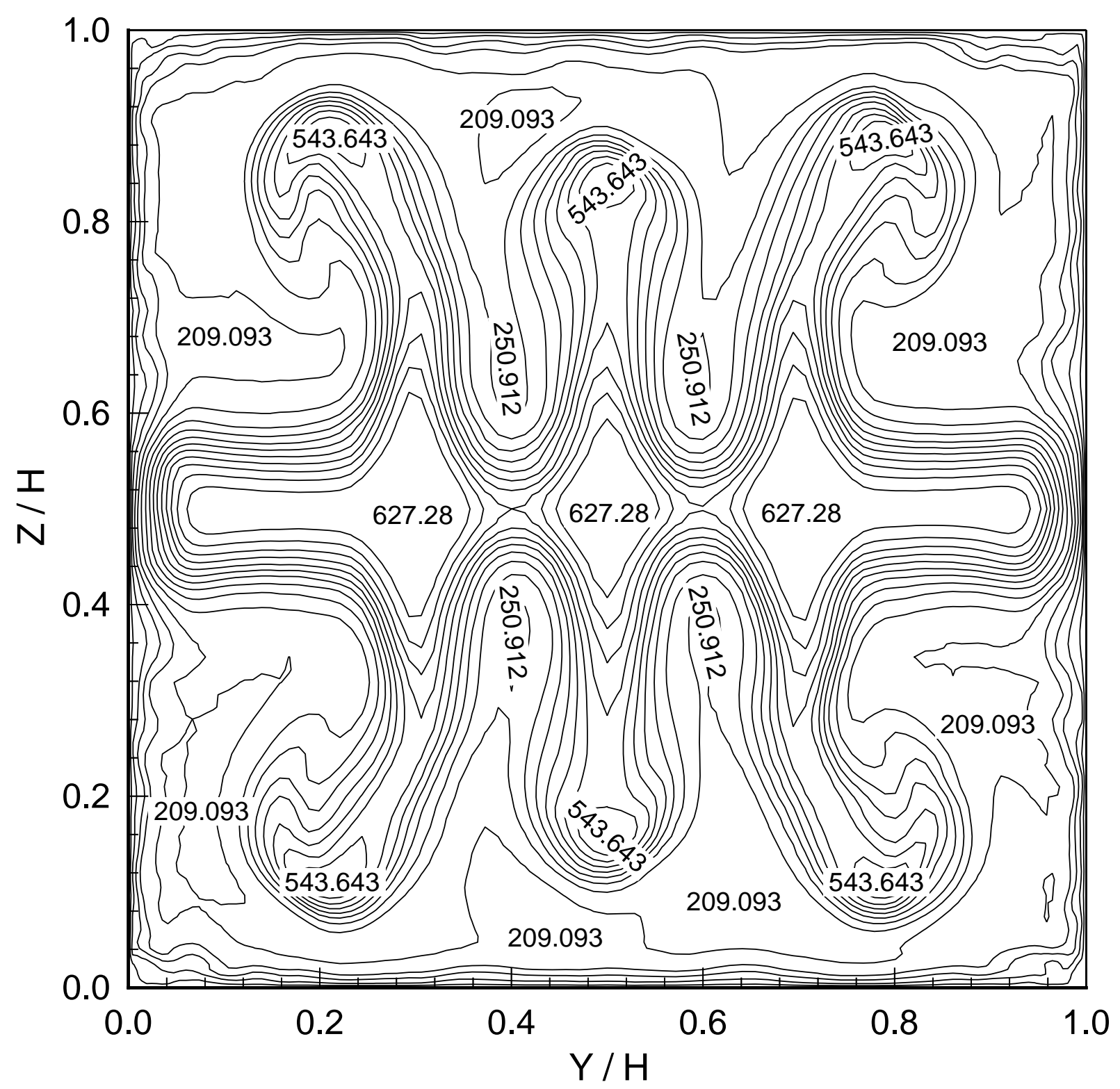

Figure 23: Contours of $U$ on the $(y, z)$ plane at $x / D=14.0$. The inflow conditions are those of Case 2 ( Table 1 ) and there are three tabs on both the upper and lower edges of the jet at $y / H=1 / 4,1 / 2$ and $3 / 4$. The vortices are of the "necklace" type resulting from a positive $V_{0}$. Contour values are in $\mathrm{m} / \mathrm{s}$. 


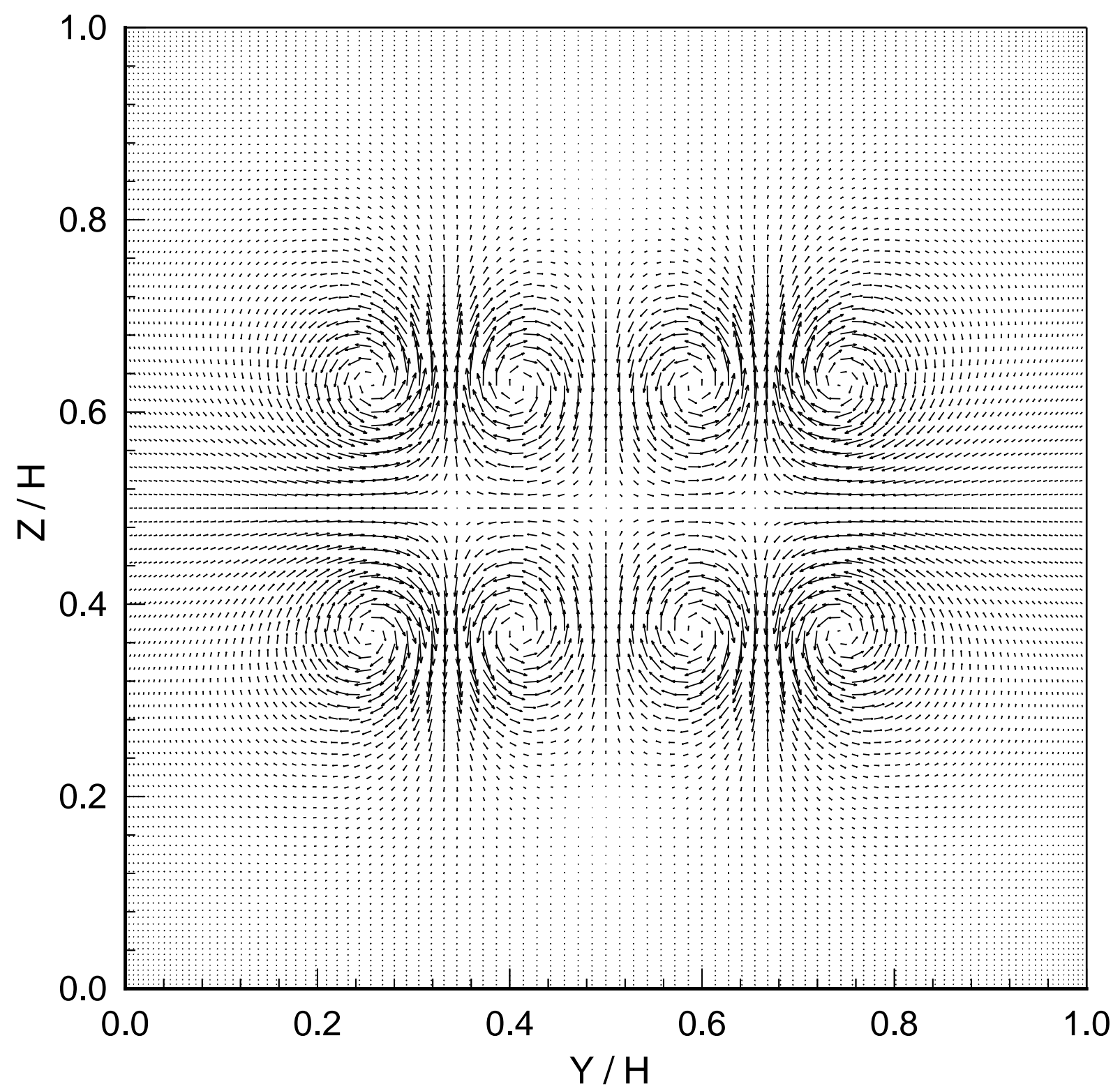

Figure 24: Vectors of $(V, W)$ on the inflow boundary, $x / D=0.0$. The maximum value of the velocity on this plane is $84.5 \mathrm{~m} / \mathrm{s}$. The inflow conditions are those of Case 6 ( Table 1 ) and there are two tabs on both the upper and lower edges of the jet at $y / H=1 / 3$ and $2 / 3$. The vortices are of the "necklace" type resulting from a positive $V_{0}$. 


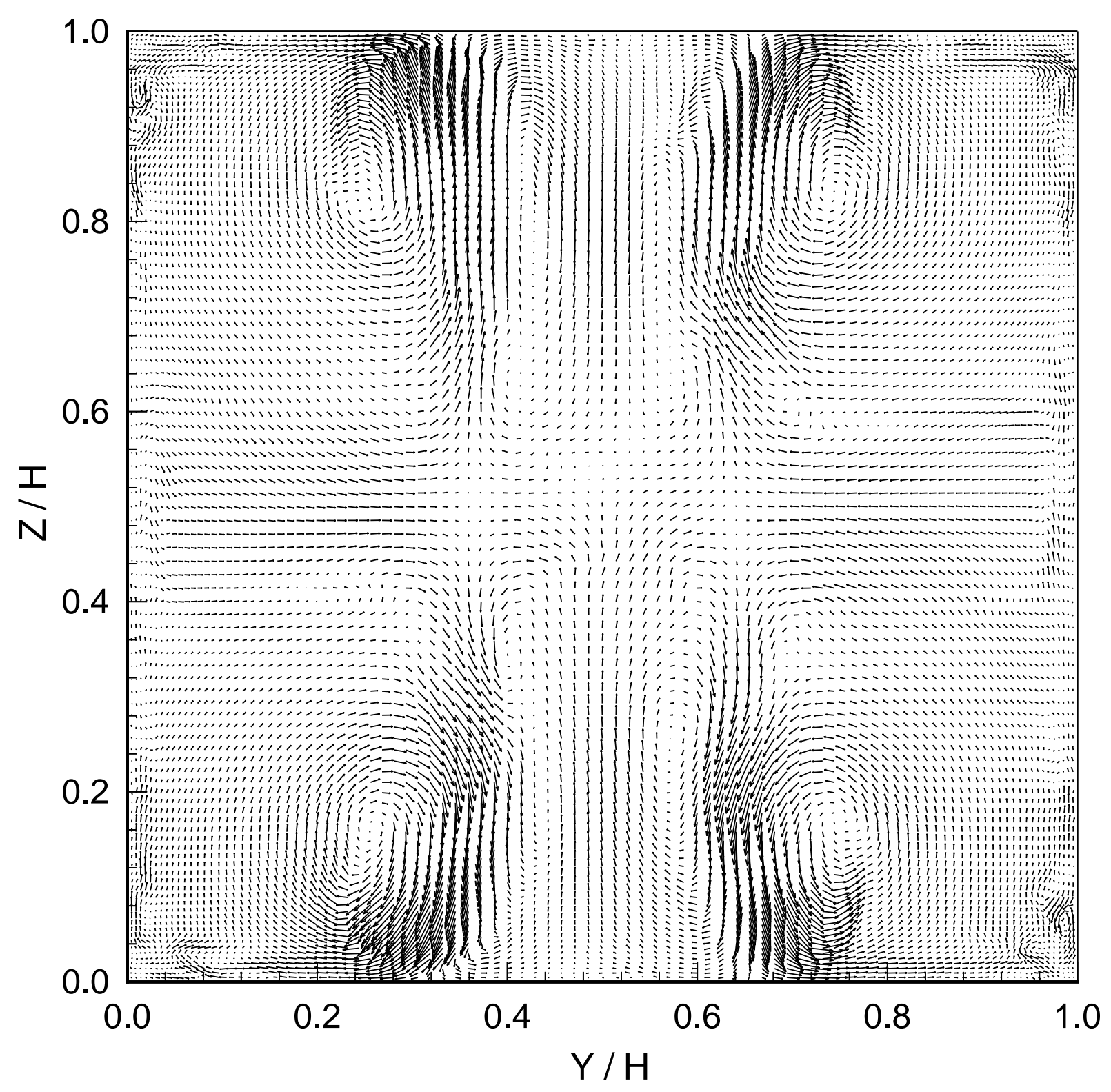

Figure 25: Vectors of $(V, W)$ on the plane at $x / D=14.0$. The maximum value of the velocity on this plane is $56.9 \mathrm{~m} / \mathrm{s}$. The inflow conditions are those of Case 6 ( Table 1 ) and there are two tabs on both the upper and lower edges of the jet at $y / H=1 / 3$ and $2 / 3$. The vortices are of the "necklace" type resulting from a positive $V_{0}$. 


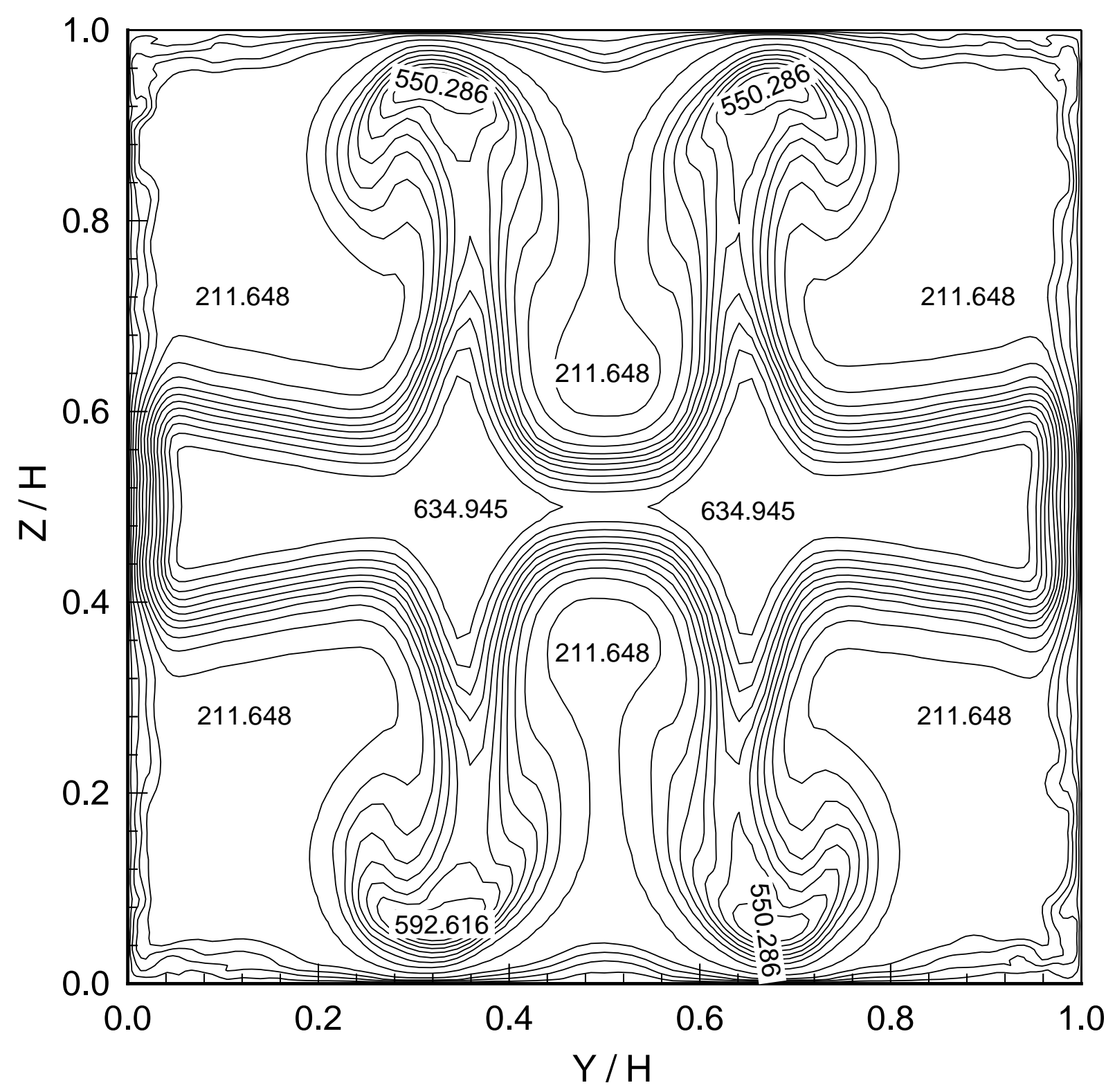

Figure 26: Contours of $U$ on the $(y, z)$ plane at $x / D=14.0$. The inflow conditions are those of Case 6 ( Table 1 ) and there are two tabs on both the upper and lower edges of the jet at $y / H=$ $1 / 3$ and $2 / 3$. The vortices are of the "necklace" type resulting from a positive $V_{0}$. Contour values are in $\mathrm{m} / \mathrm{s}$. 


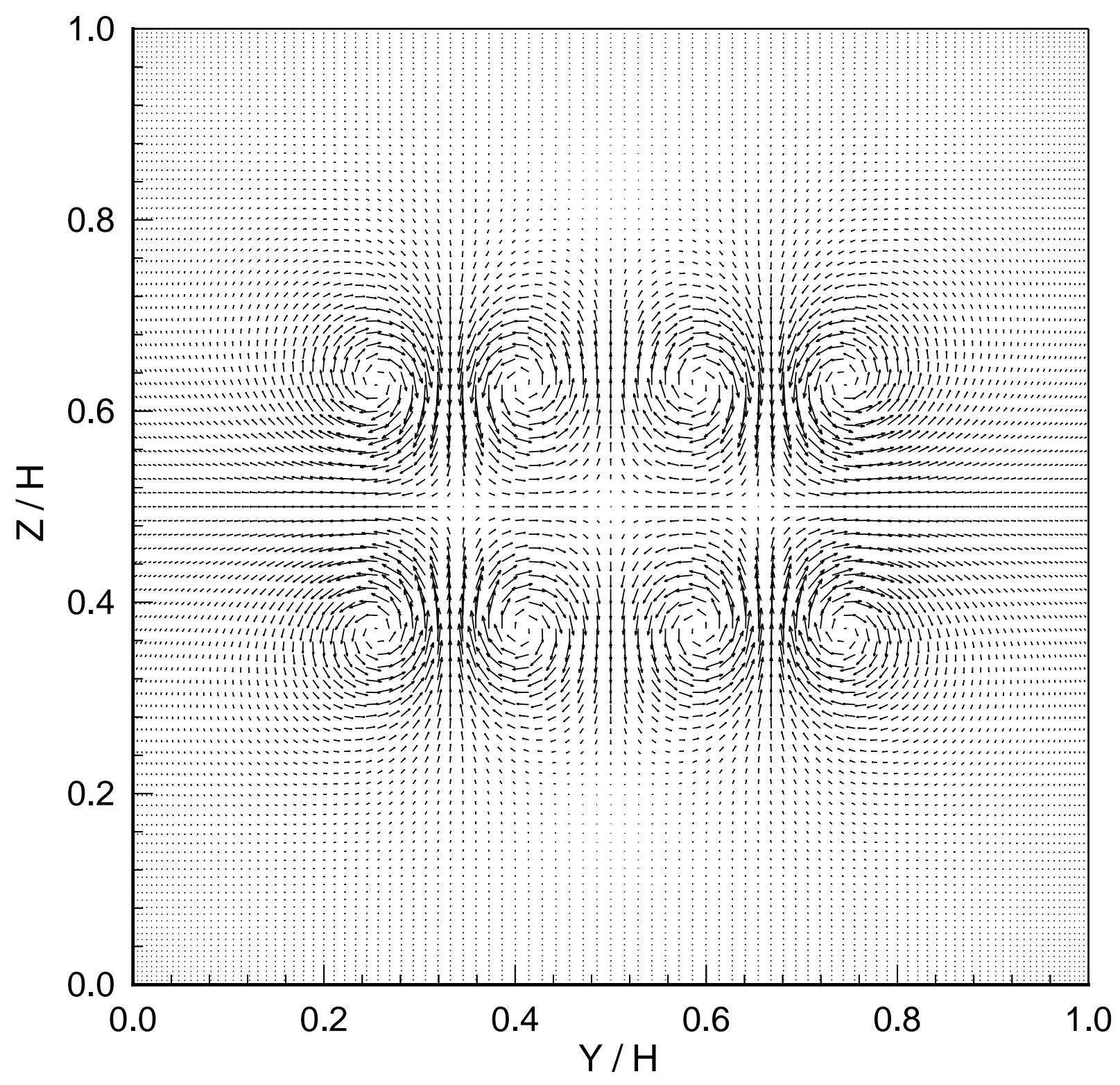

Figure 27: Vectors of $(V, W)$ on the inflow boundary, $x / D=0.0$. The maximum value of the $(V, W)$ velocity on this plane is $84.5 \mathrm{~m} / \mathrm{s}$, just as for the results shown in Figure 24. The inflow conditions are those of Case 6 ( Table 1 ) and there are two tabs on both the upper and lower edges of the jet at $y / H=1 / 3$ and $2 / 3$. The vortices are of the "trailing" type resulting from a negative $V_{0}$. 


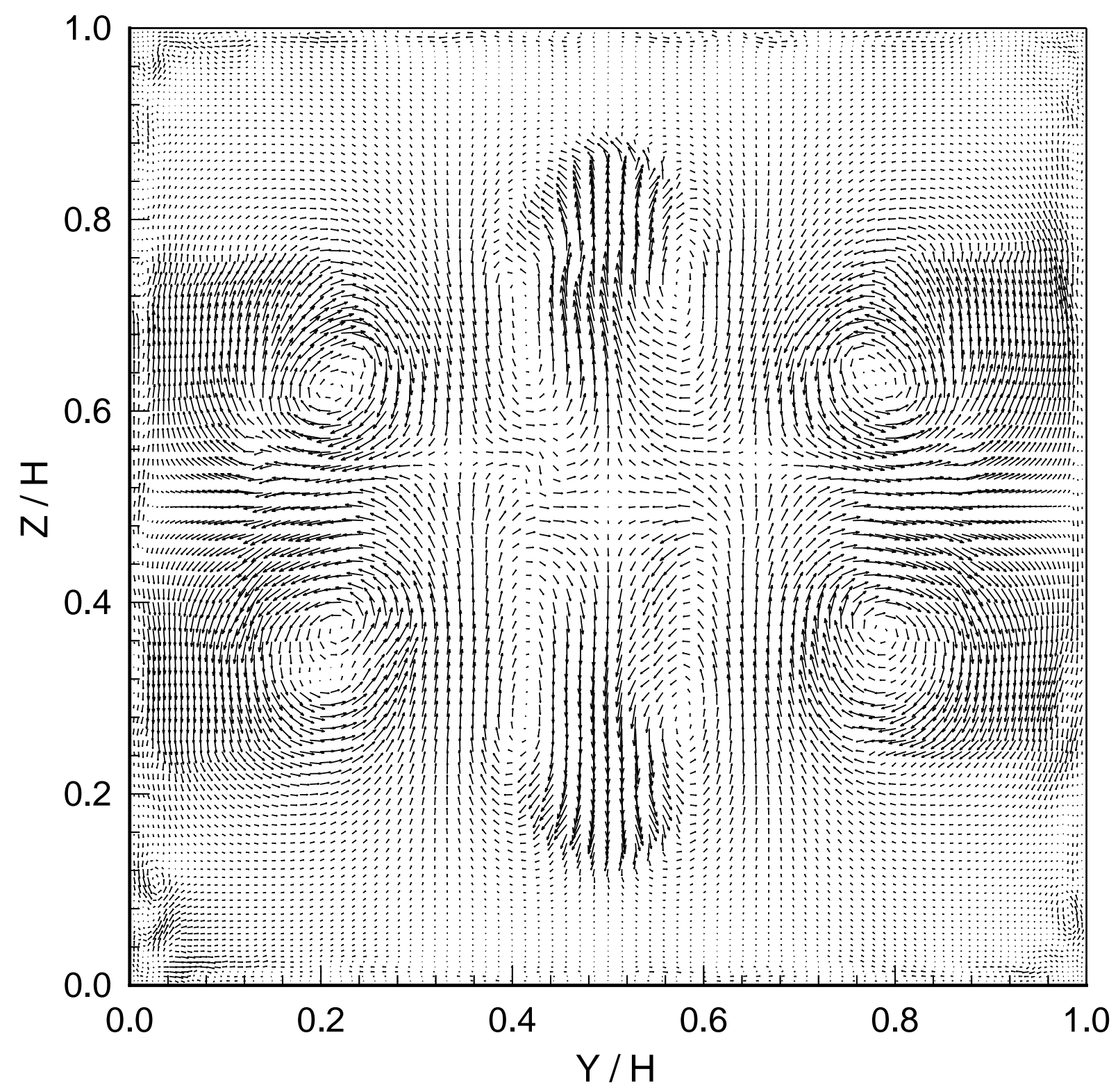

Figure 28: Vectors of $(V, W)$ on the plane at $x / D=14.0$. The maximum value of the velocity on this plane is $51.1 \mathrm{~m} / \mathrm{s}$, somewhat less than the corresponding case shown in Figure 24 . The inflow conditions are those of Case 6 ( Table 1 ) and there are two tabs on both the upper and lower edges of the jet at $y / H=1 / 3$ and $2 / 3$. The vortices are of the "trailing" type resulting from a negative $V_{0}$. 


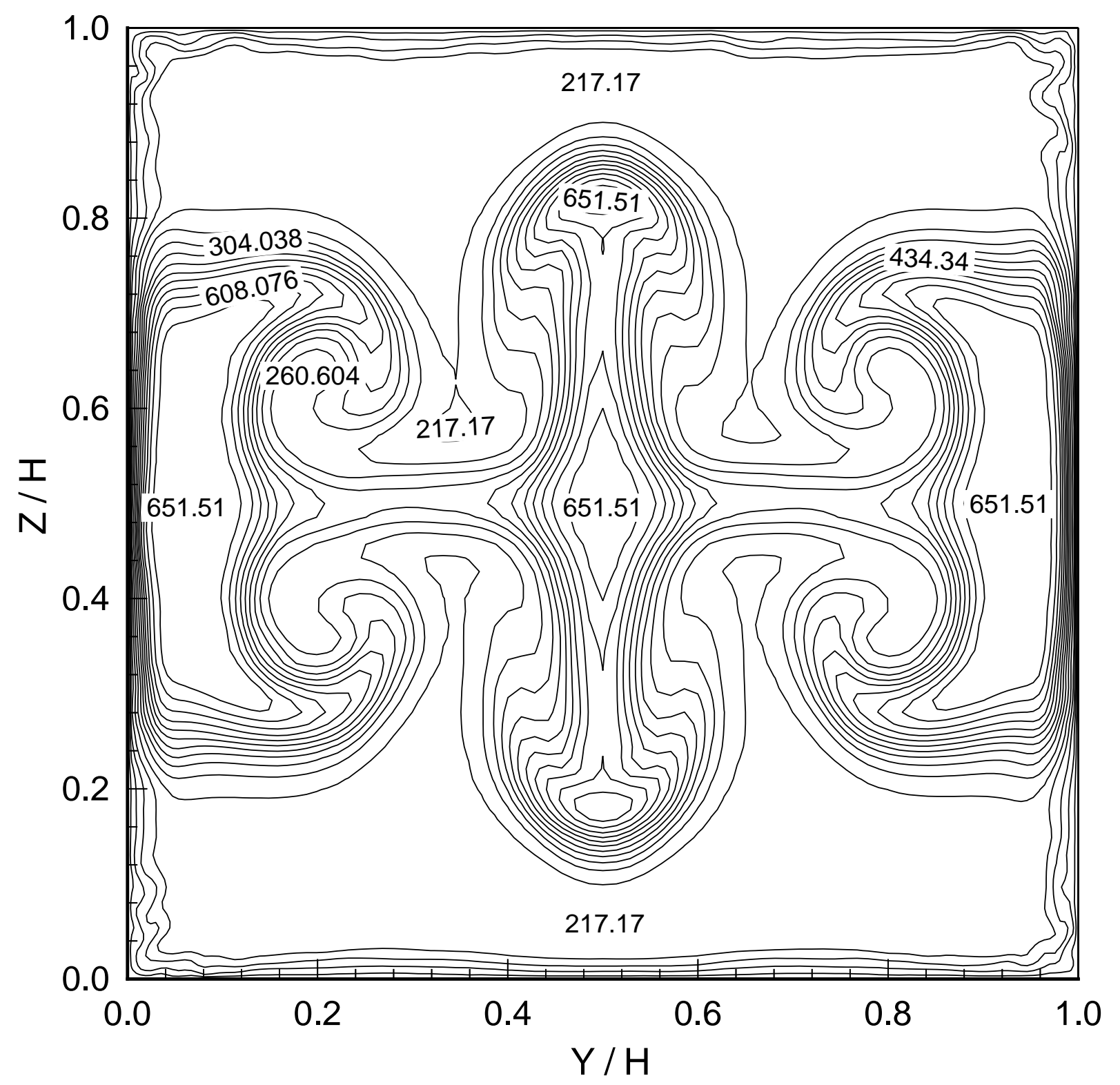

Figure 29: Contours of $U$ on the $(y, z)$ plane at $x / D=14.0$. The inflow conditions are those of Case 6 ( Table 1 ) and there are two tabs on both the upper and lower edges of the jet at $y / H=$ $1 / 3$ and $2 / 3$. The vortices are of the "trailing" type resulting from a negative $V_{0}$. Contour values are in $\mathrm{m} / \mathrm{s}$. 


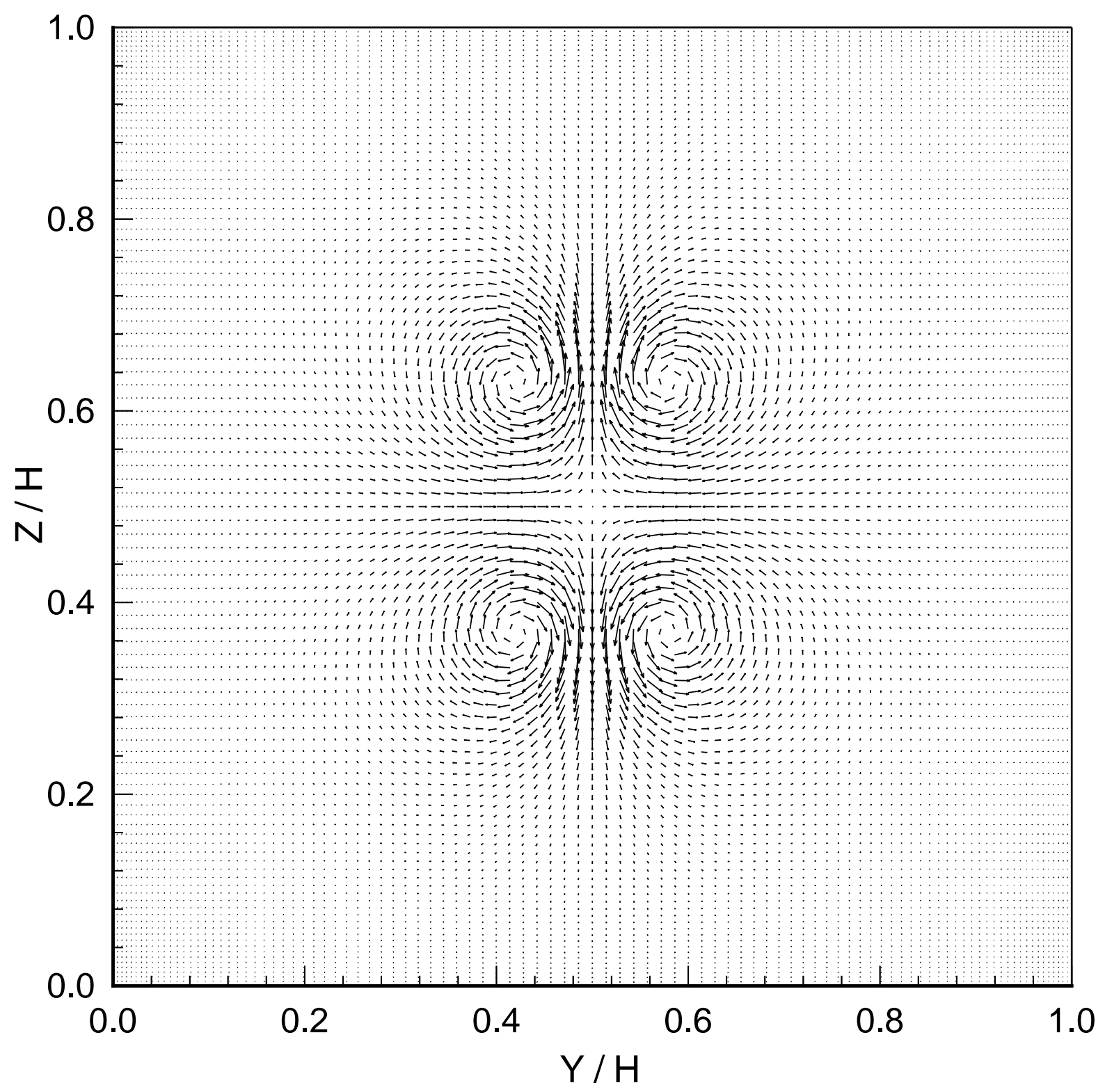

Figure 30: Vectors of $(V, W)$ on the inflow boundary, $x / D=0.0$. The maximum value of the velocity on this plane is $88.4 \mathrm{~m} / \mathrm{s}$. The inflow conditions are those of Case 7 ( Table 1 ) and there is only one tab on both the upper and lower edges of the jet at $y / H=1 / 2$. The vortices are of the "necklace" type resulting from a positive $V_{0}$. 


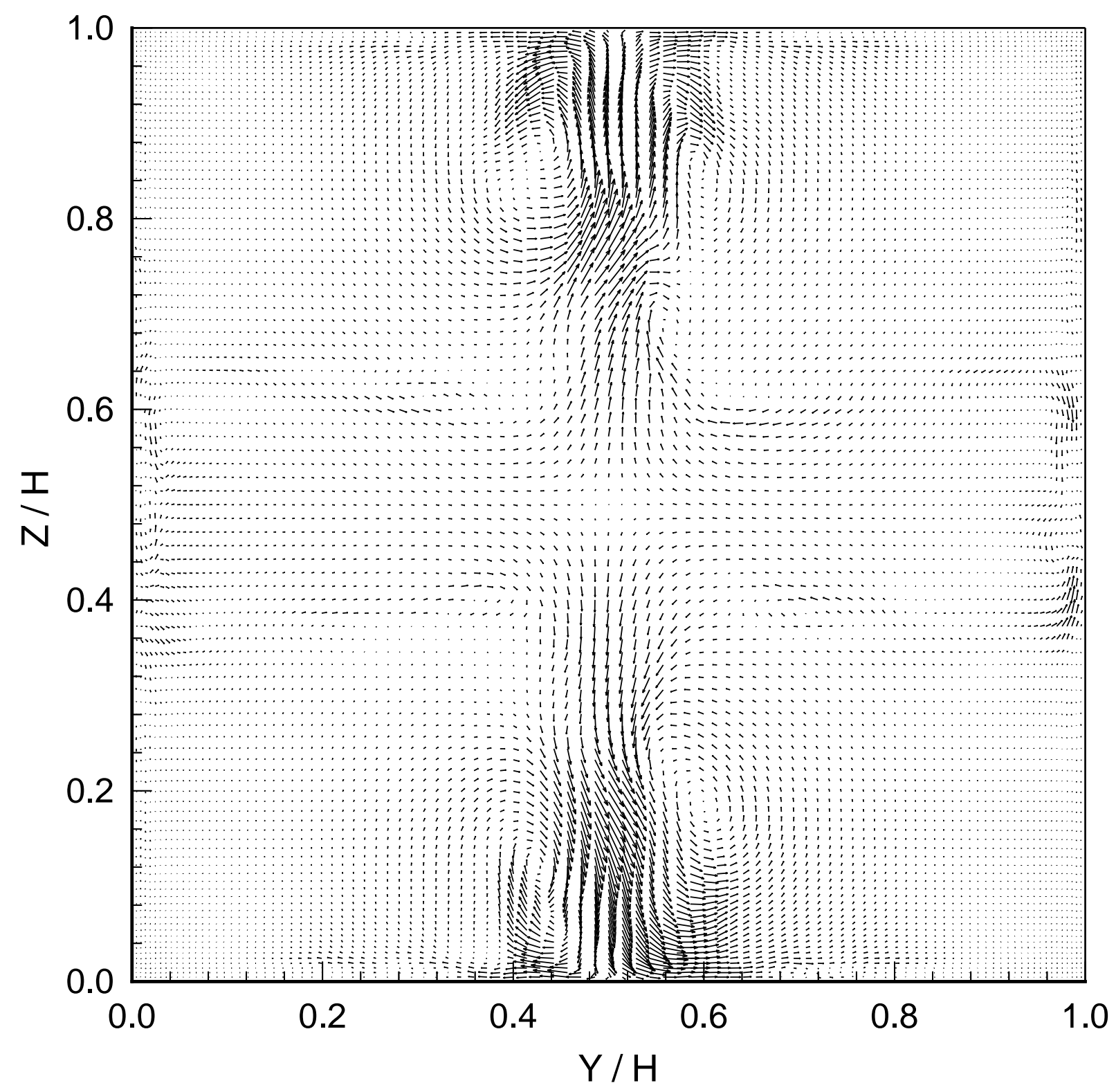

Figure 31: Vectors of $(V, W)$ on the plane at $x / D=14.0$. The maximum value of the velocity on this plane is $64.6 \mathrm{~m} / \mathrm{s}$. The inflow conditions are those of Case 7 ( Table 1 ) and there is only one tab on both the upper and lower edges of the jet at $y / H=1 / 2$. The vortices are of the "necklace" type resulting from a positive $V_{0}$. 


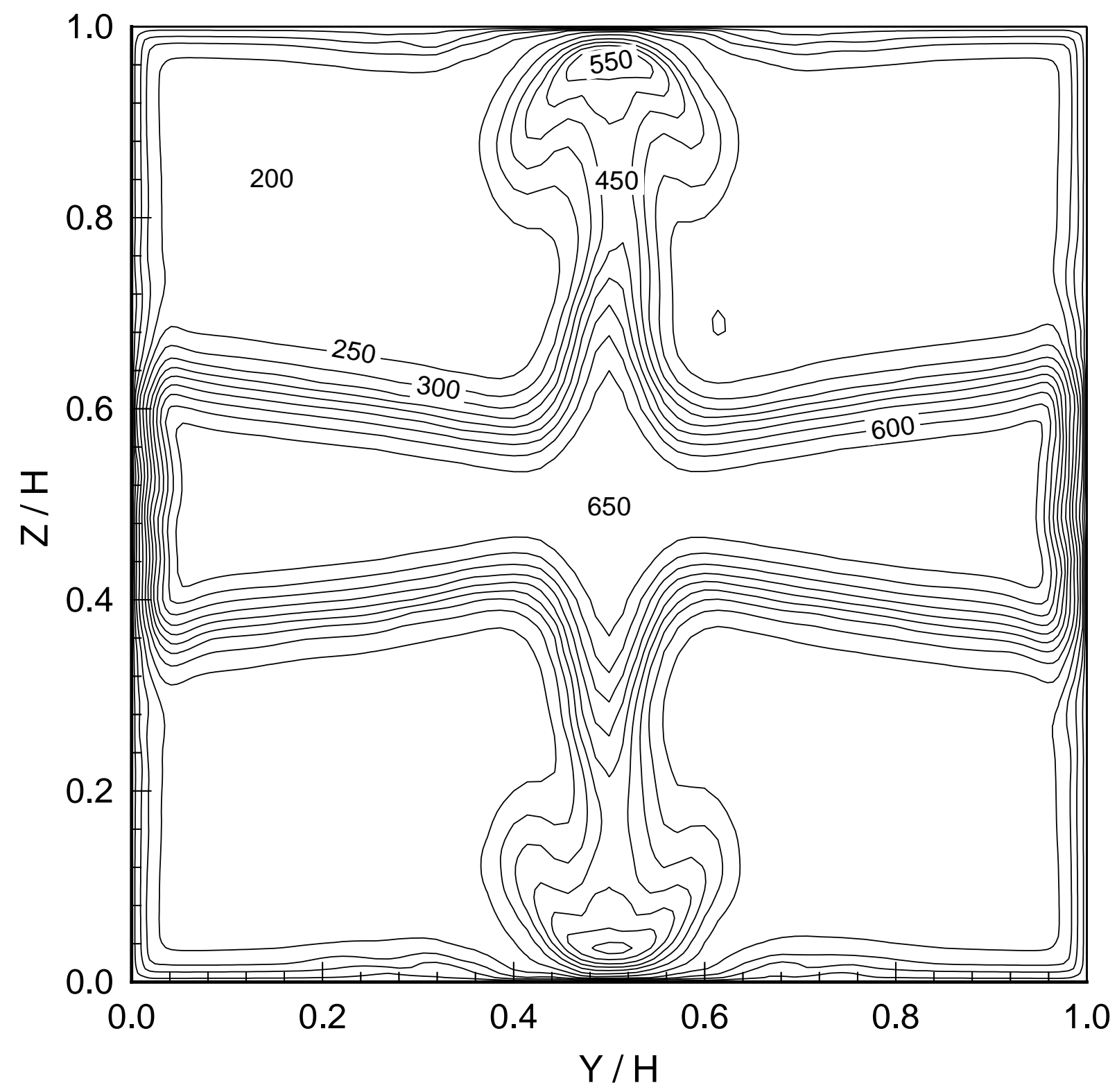

Figure 32: Contours of $U$ on the $(y, z)$ plane at $x / D=14.0$. The inflow conditions are those of Case 7 ( Table 1 ) and there is only one tab on both the upper and lower edges of the jet at $y / H=1 / 2$. The vortices are of the "necklace" type resulting from a positive $V_{0}$. Contour values are in $\mathrm{m} / \mathrm{s}$. 


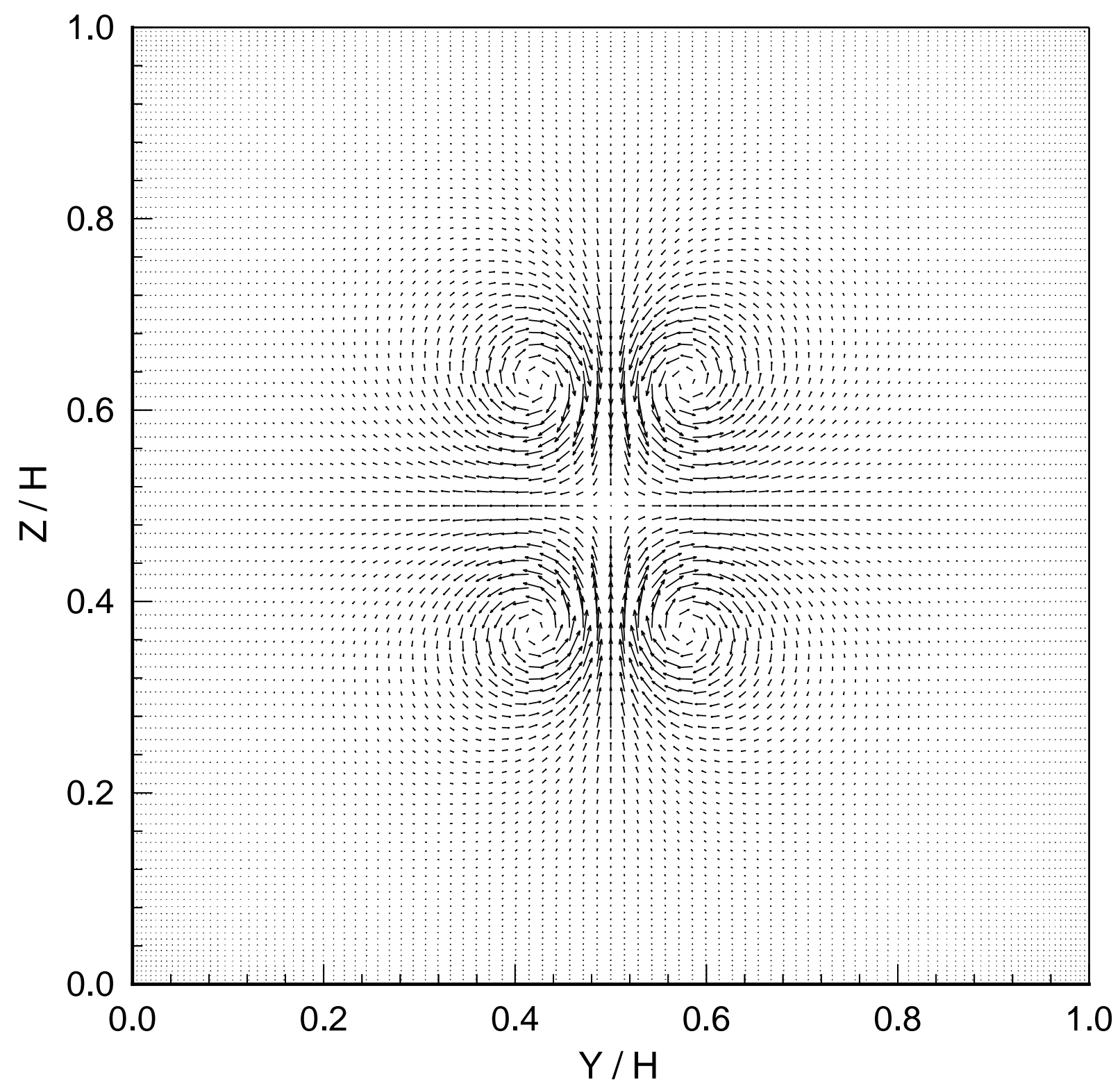

Figure 33: Vectors of $(V, W)$ on the inflow boundary, $x / D=0.0$. The maximum value of the velocity on this plane is $88.4 \mathrm{~m} / \mathrm{s}$. The inflow conditions are those of Case 7 ( Table 1 ) and there is only one tab on both the upper and lower edges of the jet at $y / H=1 / 2$. The vortices are of the "trailing" type resulting from a negative $V_{0}$. 


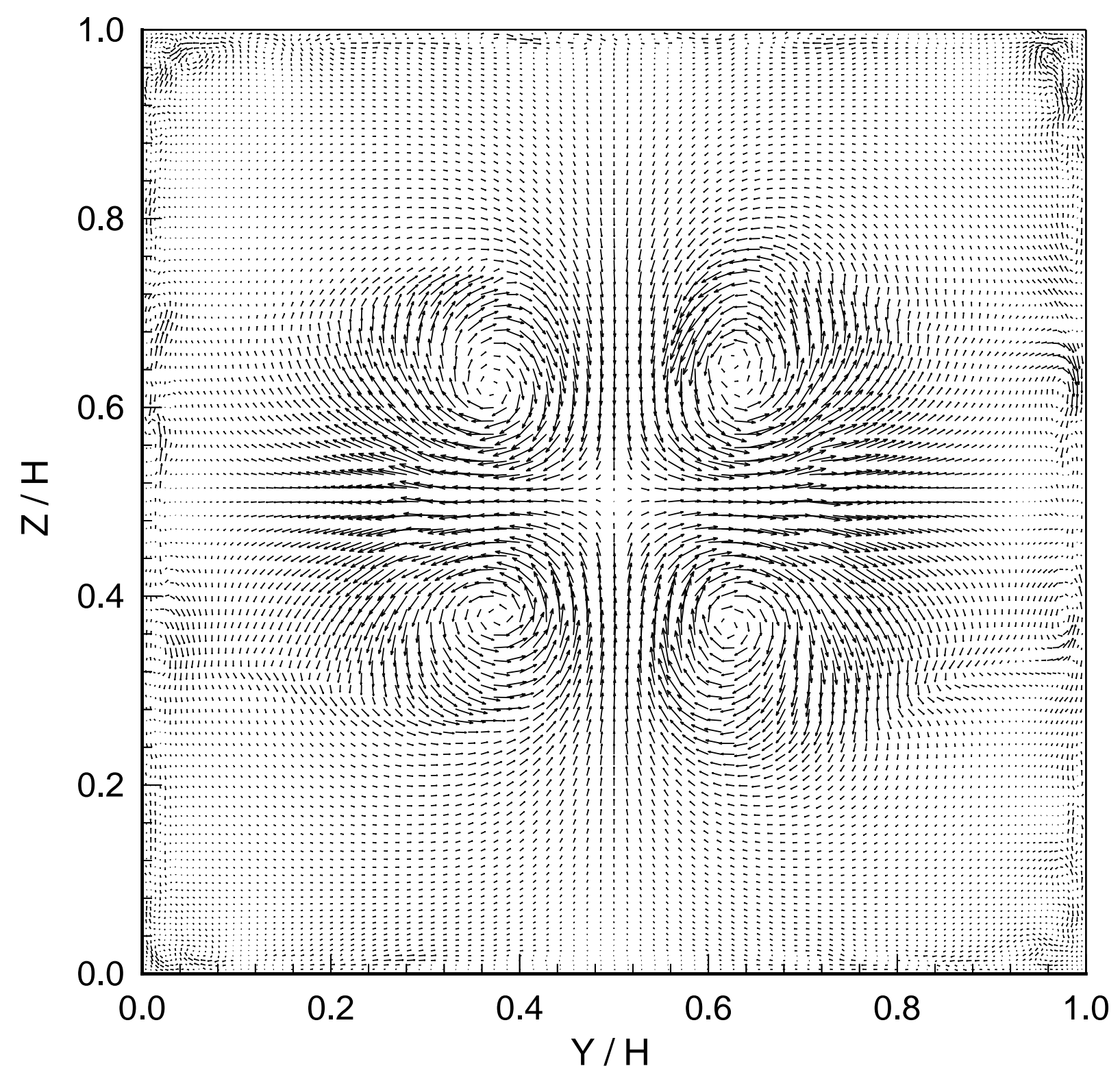

Figure 34: Vectors of $(V, W)$ on the plane at $x / D=14.0$. The maximum value of the velocity on this plane is $50.8 \mathrm{~m} / \mathrm{s}$. The inflow conditions are those of Case 7 ( Table 1 ) and there is only one tab on both the upper and lower edges of the jet at $y / H=1 / 2$. The vortices are of the "trailing" type resulting from a negative $V_{0}$. 


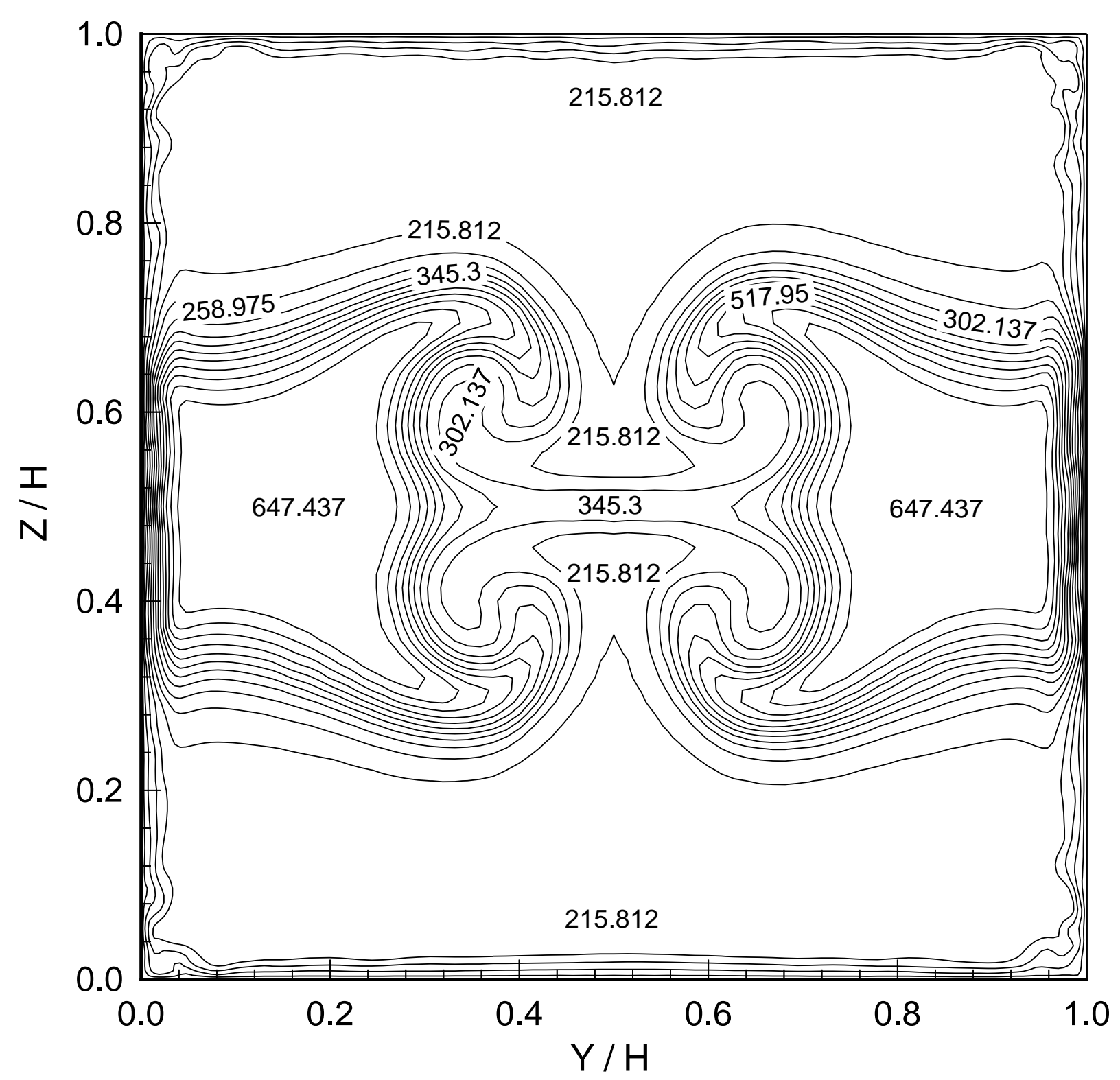

Figure 35: Contours of $U$ on the $(y, z)$ plane at $x / D=14.0$. The inflow conditions are those of Case 7 ( Table 1 ) and there is only one tab on both the upper and lower edges of the jet at $y / H=1 / 2$. The vortices are of the "trailing" type resulting from a negative $V_{0}$. Contour values are in $\mathrm{m} / \mathrm{s}$. 


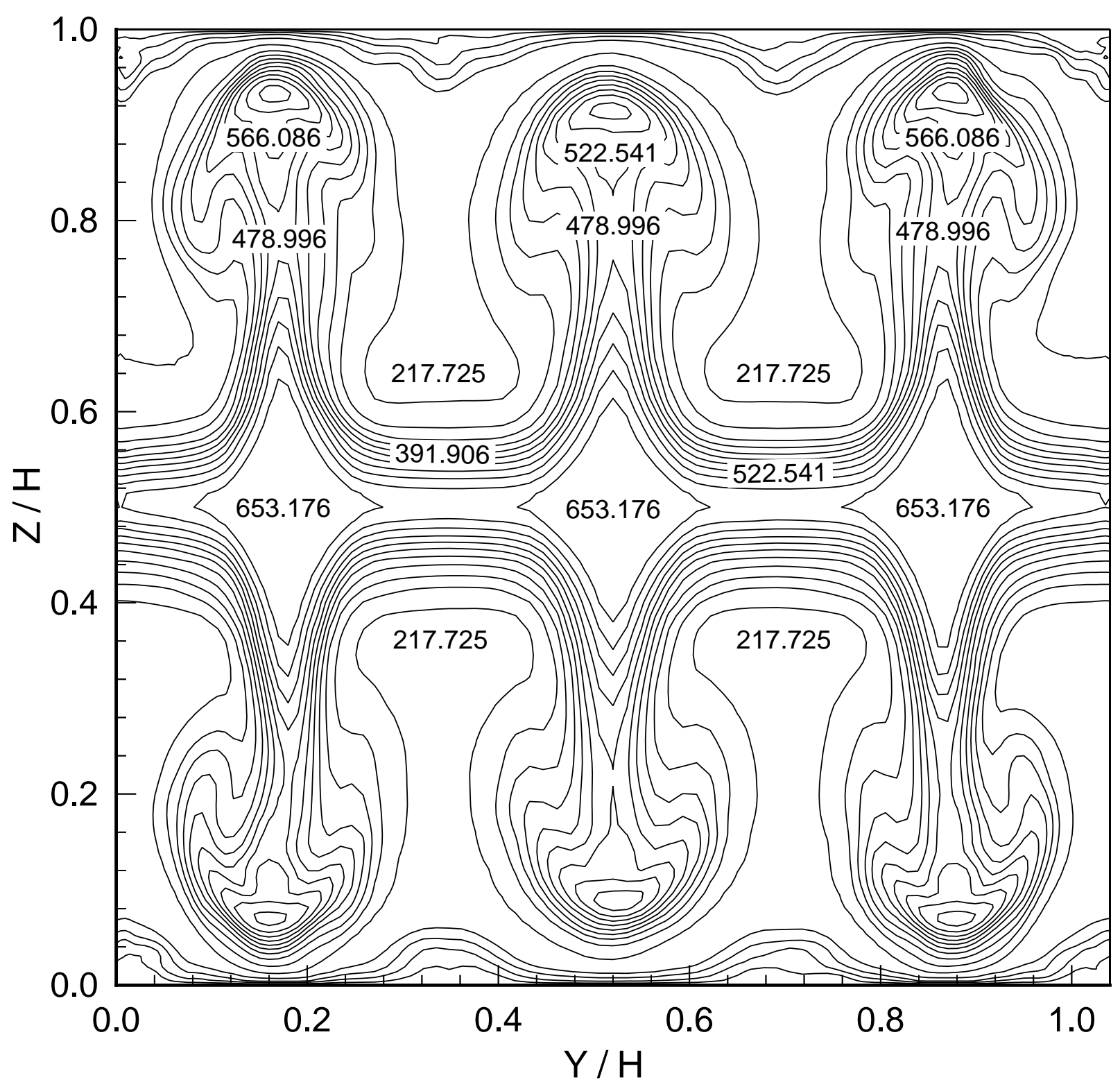

Figure 36: Contours of $U$ on the $(y, z)$ plane at $x / D=14.0$ for the case in which the side wall boundary conditions are periodic. Note that because of the periodic boundary conditions the computational domain extends from $y / H=0.0$ to $y / H=1.04$. The inflow conditions are those of Case 9 ( Table 1 ) and there are three tabs on both the upper and lower edges of the jet at $y / H=1 / 6,1 / 2$ and $5 / 6$. The vortices are of the "necklace" type resulting from a positive $V_{0}$. Contour values are in $\mathrm{m} / \mathrm{s}$. 